\title{
Performance Analysis of NOMA-based Relaying Networks with Transceiver Hardware Impairments
}

\author{
Chao Deng ${ }^{1}$, Xiaoya Zhao ${ }^{1}$, Di Zhang ${ }^{2}$, Xingwang $\mathrm{Li}^{{ }^{*}}$, Jingjing Li ${ }^{1}$ and Charles C. Cavalcante ${ }^{3}$ \\ ${ }^{1}$ School of Physics and Electronic Information Engineering, Henan Polytechnic University \\ Jiaozuo, China \\ [e-mail: super@hpu.edu.cn, zhaoxiaoya1027@163.com, lixingwangbupt@gmail.com, lijingjing_jj@sina.cn] \\ ${ }^{2}$ School of information engineering, Zhengzhou university, Zhengzhou, China \\ [e-mail:dr.di.zhang@ieee.org] \\ ${ }^{3}$ Wireless Telecommunications Research Group, Federal University of Ceará \\ Campus do Pici, Bl. 722, ZIP 60455-760, Fortaleza-CE, Brazil \\ [e-mail: charles@gtel.ufc.br] \\ *Corresponding author: Xingwang Li
}

Received December 5, 2017; revised January 26, 2018; accepted February 13, 2018; published September 30, 2018

\begin{abstract}
In this paper, the performance of non-orthogonal multiple access (NOMA) dual-hop (DH) amplify-and-forward (AF) relaying networks is investigated, where Nakagami- $m$ fading channel is considered. In order to cover more details, in our analysis, the transceiver hardware impairments at source, relay and destination nodes are comprehensively considered. To characterize the effects of hardware impairments brought in NOMA DH AF relaying networks, the analytical closed-form expressions for the exact outage probability and approximate ergodic sum rate are derived. In addition, the asymptotic analysis of the outage probability and ergodic sum rate at high signal-to-noise ratio (SNR) regime are carried out in order to further reveal the insights of the parameters for hardware impairments on the network performance. Simulation results indicate the performance of asymptotic ergodic sum rate are limited by levels of distortion noise.
\end{abstract}

Keywords: Hardware impairments, Nakagami-m fading, non-orthogonal multiple access, successive interference cancellation

This work was supported in part by the Doctoral Scientific Funds of Henan polytechnic University (no. B2016-34), in part by China Scholarship Council Grant 201608410318, in part by the Henan Scientific and Technological Research Project under Grant 182102210307, Excellent course construction project of graduate students of Henan Polytechnic University, Grant 2016YJP02. 


\section{Introduction}

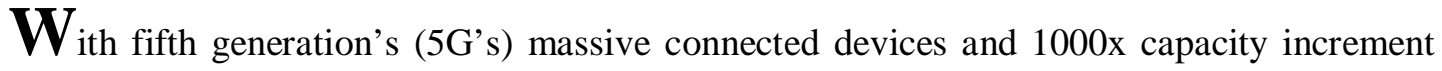
targets, the exponentially increasing data traffic is becoming a challenging issue of wireless evolution [1]. To meet this demand, a myriad of technologies have been proposed, such as multiple-input multiple-output (MIMO) [2] [3], ultra-dense network (UDN) [4], small cell network (SCN) [5], cooperative cellular network [6], and non-orthogonal multiple access (NOMA) [7]. Recently, NOMA has received a great deal of interests from both academia and industry since it has the potential to improve spectral efficiency. In contrast to the traditional orthogonal multiple access (OMA), NOMA can simultaneously accommodate a plurality of users to multiplex within the same time and frequency resources by allocating different power to different user based on the channel conditions. Afterwards, the successive interference cancellation (SIC) is executed at the receiver side to decode packets that arrive simultaneously [8] [9].

In literature, a great deal of research contributions in the fields of NOMA based on fading channels have been established in [10]-[16]. The performance of the cooperative NOMA relaying networks over independent Rayleigh fading channels was investigated in [10] and [11], where a suboptimal power allocation scheme for NOMA used at the source was proposed. Authors in [12] provided the analytical expressions for the outage probability and ergodic sum rate over frequency-flat block-fading channels, and it is shown that the proposed NOMA scheme provides remarkable performance gain compared with conventional ones. The closed-form expressions and asymptotic approximations for the ergodic sum rate and outage probability over independent Rayleigh fading channels were analyzed in [13]. In [14], the exact closed-form expressions for outage probability and throughput of NOMA-based dual-hop (DH) amplify-and-forward (AF) fixed gain relaying networks over Nakagami- $m$ fading channels were derived. Authors in [15] derived the closed-form expressions on upper and lower bounds for the outage probability and ergodic sum rate of NOMA-based DH AF relaying networks over Nakagami- $m$ fading channels. Considering imperfect channel state information (CSI), [16] presented closed-form expressions on the exact and tight lower bounds for the outage probability of NOMA-based downlink AF relaying networks over Nakagami- $m$ fading channels. However, all those previous studies are built on the ideal hardware conditions. In practice, communication networks suffer from hardware impairments caused by phase noise, in-phase/quadrature- phase (I/Q) imbalance, high power amplifier non-linearities, and quantization errors [17]. Although the impact of these hardware impairments may be somehow mitigated by using compensation algorithms and calibration methods, they cannot be completely removed due to the estimation error, inaccurate calibration methods and different types of noise [18]. Therefore, the hardware impairments cannot be simply ignored.

The aforementioned literature lays a solid foundation in terms of NOMA-based relaying networks with ideal radio frequency (RF). However, the impact of hardware impairments on the performance of NOMA relaying networks in terms of transceiver hardware impairments is not considered yet. Motivated by the previous discussion, we herein try to bridge this gap by exploring the performance of NOMA-based relaying networks in presence of hardware impairments, where Nakagami- $m$ fading channel is considered since it is a versatile model that can be widely used to describe various fading channels, such as the Gaussian channel, Rayleigh channel and Rician channel, etc. In this NOMA DH AF relaying networks, the source transmits signals to far user through a relaying node, and there is no direct link between 
the source and far receiver due to the severe shadowing fading and path loss. In contrast, the near user can receive the signals from both the source and relay. More particularly, we derive exact and approximate expressions for the outage probability and ergodic sum rate of NOMA relaying networks in the presence of aggregated transceiver hardware impairments. To get more insights, the asymptotic analyses for the outage probability and the ergodic sum rate are performed. The primary contributions of this paper are summarised as follows:

1) Considering hardware impairments and Nakagami-m fading channels, we derive the exact analytical closed-form expressions of outage probability for the far and near users. In order to obtain more useful insights, the asymptotic expressions of the outage probability for the two users are derived.

2) We investigate the ergodic sum rate performance of NOMA relaying networks in the presence of hardware impairments by deriving the approximate expression for the ergodic sum rate of NOMA relaying networks. We further pursue the asymptotic analysis for the ergodic sum rate and derive the asymptotic expression for the ergodic sum rate of NOMA relaying network over Nakagami-m fading channels. For ideal condition, the analytical upper bound of ergodic sum rate is derived. It indicates that in the case of hardware impairments, the ergodic sum rate approaches to a constant value as the average signal-to-noise ratio (SNR) increasing.

The remainder of this paper is organized as follows: Section 2 describes the NOMA relaying network model with impaired hardware. In Section 3, the exact and asymptotic expressions of outage probability for the far and near users are derived in closed-form. In Section 4, the approximate analytical expression of the ergodic sum rate is derived, followed by our asymptotic analysis for impaired hardware and upper bound for ideal hardware. Numerical results are presented in Section 5 before we concluding the paper in Section 6 .

\section{NOMA Relaying Networks Model}

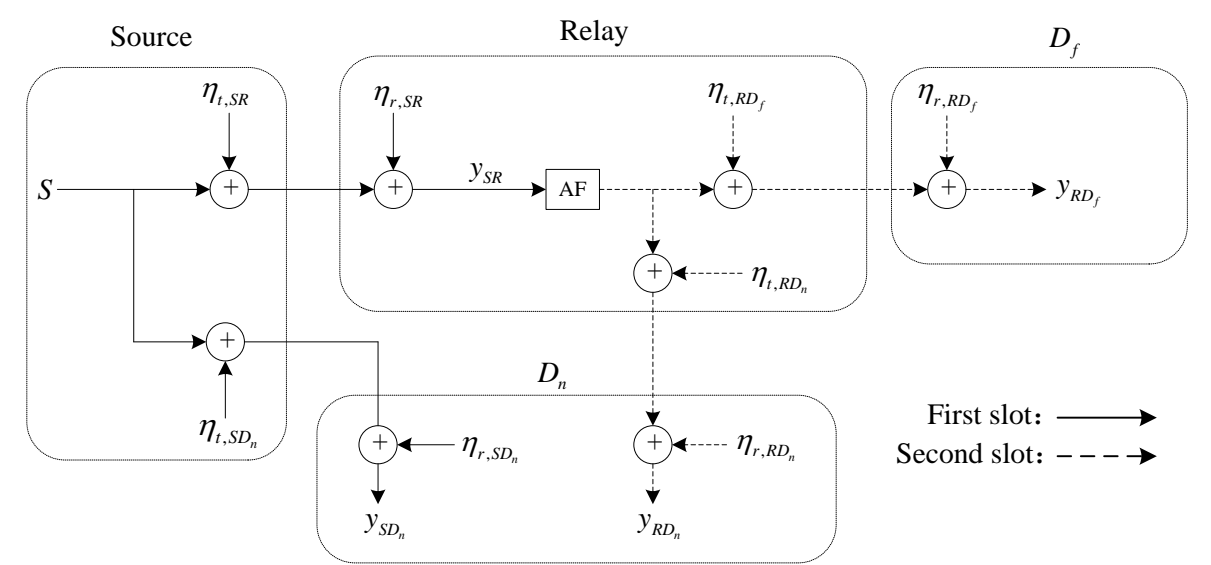

Fig. 1. System model of NOMA AF relaying network with transceiver hardware impairments

In this work, we consider a NOMA DH AF relaying network that depicted in Fig. 1. The network consists of a source $(S)$, a relay $(R)$, a far user $\left(D_{f}\right)$ and a near user $\left(D_{n}\right)$, where all nodes are equipped with a single antenna, and $D_{f}$ and $D_{n}$ are paired together for transmission in the same resource slot using the NOMA protocol. We assume the direct link between $S$ and 
$D_{n}$ can be established, while the direct link between the $S$ and $D_{f}$ is impossible due to obstacles and severe shadowing. The channel coefficients of $S$-to- $R, R$-to- $D_{f}, R$-to- $D_{n}$ and $S$-to- $D_{n}$ links are denoted as $h_{S R}, h_{R D_{f}}, h_{R D_{n}}, h_{S D_{n}}$, respectively. The transmission powers of $S$ and $R$ are $\mathrm{P}_{S}$ and $\mathrm{P}_{R}$. For convenience, we assume $\mathrm{P}_{S}=\mathrm{P}_{R}=\mathrm{P}$. Meanwhile, the additive white Gaussian noise (AWGN) in terms of all the links has zero mean and variance $N_{0}$ [14]. The communication processes are segregated into two time slots.

The first time slot: leveraging the NOMA protocol, $S$ sends $\sqrt{a_{f} \mathrm{P}_{s}} s_{f}+\sqrt{a_{n} \mathrm{P}_{s}} s_{n}$ to $R$ and $D_{n}$, where $a_{f}$ and $a_{n}$ are the power allocation coefficients satisfying with $a_{f}>a_{n}$ and $a_{f}+a_{n}=1$. Meanwhile, $s_{f}$ and $s_{n}$ are the messages sending to $D_{f}$ and $D_{n}$ with $\mathrm{E}\left[\left|s_{f}\right|^{2}\right]=\mathrm{E}\left[\left|s_{n}\right|^{2}\right]=1$, respectively. Thus, the received signals at $R$ and $D_{n}$ are expressed as

$$
y_{i}=h_{i}\left(\sqrt{a_{f} \mathrm{P}_{s}} s_{f}+\sqrt{a_{n} \mathrm{P}_{s}} s_{n}+\eta_{t, i}\right)+\eta_{r, i}+v_{i}, i=\left\{S R, S D_{n}\right\}
$$

where $\eta_{t, i}$ and $\eta_{r, i}$ are distortion noises from the transmitter and receiver, respectively [19]-[21]. As stated [21], the distortion noises are termed as

$$
\eta_{t, i} \sim C N\left(0, \kappa_{t, i}^{2} \mathrm{P}\right), \eta_{r, i} \sim C N\left(0, \boldsymbol{\kappa}_{r, i}^{2} \mathrm{P}\left|h_{i}\right|^{2}\right)
$$

where the design parameters $\kappa_{t, i}, \kappa_{r, i} \geq 0$ characterize the level of impairments at the transmitter and receiver, respectively. These parameters can be construed as error vector magnitude (EVM) [22]. As seen from (2), the impairment model in (1) is equivalent to

$$
y_{i}=h_{i}\left(\sqrt{a_{f} \mathrm{P}_{S}} s_{f}+\sqrt{a_{n} \mathrm{P}_{S}} s_{n}+\eta_{i}\right)+v_{i}, i=\left\{S R, S D_{n}\right\}
$$

where the independent distortion noise obeys $\eta_{i} \sim C N\left(0, \kappa_{\mathrm{i}}^{2} \mathrm{P}\right)$, and it can be used to model the hardware impairments from both transmitter and receiver with definition $\kappa_{i}=\sqrt{\kappa_{t, i}^{2}+\kappa_{r, i}^{2}}$. Note that (3) reduces to be the ideal hardware expression for $\kappa_{i}=0, i=S R, S D_{n}$, thus the ideal system is given by

$$
y_{i}=h_{i}\left(\sqrt{a_{f} \mathrm{P}_{S}} s_{f}+\sqrt{a_{n} \mathrm{P}_{S}} s_{n}\right)+v_{i}, i=\left\{S R, S D_{n}\right\}
$$

where $v_{i}$ are AWGN at the relay and $D_{n}$, respectively. Meanwhile, $h_{i}$ follows Nakagami-m distribution, such that the channel gains $\rho_{i}=\left|h_{i}\right|^{2} \sim G\left(\alpha_{i}, \beta_{i}\right)$. Therefore, the probability density function (PDF) and the cumulative density function (CDF) of the channel gains $\rho_{i}$ are expressed as

$$
\begin{gathered}
f_{\rho_{i}}(x)=\frac{x^{\alpha_{i}-1}}{\Gamma\left(\alpha_{i}\right) \beta_{i}^{\alpha_{i}}} e^{-x / \beta_{i}}, x \geq 0 \\
F_{\rho_{i}}(x)=1-\sum_{g_{i}=0}^{\alpha_{i}-1} \frac{e^{-x / \beta_{i}}}{g_{i} !}\left(\frac{x}{\beta_{i}}\right)^{g_{i}}, x \geq 0
\end{gathered}
$$

where $\Gamma\left(\alpha_{i}\right)$ denotes the Gamma function [16]. The received signal-to-interference-plus-noise ratio (SINR) for $D_{n}$ to decode $D_{f}$ 's signal is given by

$$
\tilde{\gamma}_{S D_{f \rightarrow n}}=\frac{a_{f} \gamma \rho_{S D_{n}}}{\left(a_{n}+\kappa_{S D_{n}}^{2}\right) \gamma \rho_{S D_{n}}+1}
$$


where $\gamma=\mathrm{P}_{s} / N_{0}$ is the transmit average SNR at $S$. Therefore, the far user's message $s_{f}$ will be removed from the observation of $D_{n}$ if it can be successfully decoded. If message $s_{n}$ can be decoded successfully, then the received SINR at $D_{n}$ will be

$$
\tilde{\gamma}_{S D_{n}}=\frac{a_{n} \gamma \rho_{S D_{n}}}{\kappa_{S D_{n}}^{2} \gamma \rho_{S D_{n}}+1}
$$

The second time slot: the relay amplifies and forwards the received signal to the destination. Thus the received signal at $D_{f}$ and $D_{n}$ can be expressed as

$$
y_{i}=G h_{i}\left(h_{S R} \sqrt{a_{f} \mathrm{P}_{S}} s_{f}+h_{S R} \sqrt{a_{n} \mathrm{P}_{S}} s_{n}+h_{S R} \eta_{S R}+v_{S R}\right)+h_{i} \eta_{i}+v_{i}, i=\left\{R D_{f}, R D_{n}\right\}
$$

where ${ }_{G}=\sqrt{\left.\mathrm{P}_{R} /\left(\mathrm{P}_{S} \mid h_{S R}\right)^{2}\left(1+\kappa_{S R}^{2}\right)+N_{0}\right)}$ is the amplifying gain factor, $\eta_{i} \sim C N\left(0, \kappa_{\mathrm{i}}^{2} \mathrm{P}\right)$ is the distortion noise at the user $D_{f}$ or $D_{n}, v_{i}$ represents the complex Gaussian receiver noise. When $\kappa_{i}=0$, (9) reduces to the ideal system as

$$
y_{i}=G h_{i}\left(h_{S R} \sqrt{a_{f} \mathrm{P}_{S}} s_{f}+h_{S R} \sqrt{a_{n} \mathrm{P}_{S}} s_{n}+v_{S R}\right)+v_{i}, \quad i=\left\{R D_{f}, R D_{n}\right\}
$$

For non-ideal conditions, the received SINR of the user $D_{f}$ is given by

$$
\tilde{\gamma}_{R D_{f}}=\frac{a_{f} \gamma^{2} \rho_{S R} \rho_{R D_{f}}}{\left(a_{n}+d_{1}\right) \gamma^{2} \rho_{S R} \rho_{R D_{f}}+b_{1} \gamma \rho_{S R}+b_{2} \gamma \rho_{R D_{f}}+1}
$$

where $d_{1}=\kappa_{S R}^{2}+\kappa_{R D_{f}}^{2}+\kappa_{S R}^{2} \kappa_{R D_{f}}^{2}, b_{1}=1+\kappa_{S R}^{2}, b_{2}=1+\kappa_{R D_{f}}^{2}$, the Eq. (11) consists of four components: (a) the desired user $D_{f}$ 's signal component $a_{f} \gamma^{2} \rho_{S R} \rho_{R D_{f}}$, (b) the interference from user $D_{n}$ 's signal $a_{n} \gamma^{2} \rho_{S R} \rho_{R D_{f}}+\gamma \rho_{S R}+\gamma \rho_{R D_{f}} \quad$, (c) the hardware impairments component $d_{1} \gamma^{2} \rho_{S R} \rho_{R D_{f}}+\kappa_{S R}^{2} \gamma \rho_{S R}+\kappa_{R D_{f}}^{2} \gamma \rho_{R D_{f}}$ and (d) the normalized AWGN. We assume that perfect SIC is employed at $D_{f}$ and $D_{n}$, then the SINRs for the two users are expressed as

$$
\begin{gathered}
\tilde{\gamma}_{R D_{f \rightarrow n}}=\frac{a_{f} \gamma^{2} \rho_{S R} \rho_{R D_{n}}}{\left(a_{n}+d_{2}\right) \gamma^{2} \rho_{S R} \rho_{R D_{n}}+b_{1} \gamma \rho_{S R}+b_{3} \gamma \rho_{R D_{n}}+1} \\
\tilde{\gamma}_{R D_{n}}=\frac{a_{n} \gamma^{2} \rho_{S R} \rho_{R D_{n}}}{d_{2} \gamma^{2} \rho_{S R} \rho_{R D_{n}}+b_{1} \gamma \rho_{S R}+b_{3} \gamma \rho_{R D_{n}}+1}
\end{gathered}
$$

where $d_{2}=\kappa_{S R}^{2}+\kappa_{R D_{n}}^{2}+\kappa_{S R}^{2} \kappa_{R D_{n}}^{2}, b_{3}=1+\kappa_{R D_{n}}^{2}$. Note that (7), (8) and (11)-(13) reduce to be the ideal hardware expressions of SINRs as $\kappa_{S R}=\kappa_{R D_{f}}=\kappa_{R D_{n}}=\kappa_{S D_{n}}=0$. Finally, the selection combining (SC) algorithm is employed at $D_{n}$.

\section{Outage Probability Analysis}

In this section, the outage probability and the asymptotic performance for the two users of NOMA relaying networks over Nakagami- $m$ fading channel are investigated. Furthermore, regardless of non-ideal/ideal hardware, the method of outage probability is the same, but the difference is that when the aggregate level of impairments $\kappa_{S R}=\kappa_{R D_{n}}=\kappa_{R D_{f}}=\kappa_{S D_{n}}=0$, the outage probability expressions simplify into the ideal ones at $D_{n}$ and $D_{f}$ on ideal system. Therefore, we are not going to elaborate on the classical process from the special case of ideal hardware in this section. 


\subsection{Exact Outage Behavior}

This section derives the closed-form expressions for the exact outage probability, so the outage probability at $D_{f}$ and $D_{n}$ are denoted by $\mathrm{P}_{\text {out }}^{D_{f}}$ and $\mathrm{P}_{\text {out }}^{D_{n}}$, respectively. In addition, we consider that the target SINRs of the two users are determined by their channel condition. In the following, the outage probability is derived for the two time slots over Nakagami- $m$ fading.

1) Outage Probability for $D_{f}$

The outage events will occur at $D_{f}$ if $D_{f}$ fails to decode its own signal. In this case, the outage probability at $D_{f}$ can be expressed as

$$
\mathrm{P}_{\text {out }}^{D_{f}}=\operatorname{Pr}\left(\tilde{\gamma}_{R D_{f}}<\gamma_{\text {thf }}\right)
$$

where $\gamma_{\text {thf }}$ denotes the outage threshold at $D_{f}$.

The following theorem provides new closed-form expressions for $D_{f}$ in the presence of transceiver hardware impairments.

Theorem 1: For Nakagami- $m$ fading channels, the exact closed-form expression for the outage probability of $D_{f}$ in presence of hardware impairments is given as

- Non-ideal condition $\left(\kappa_{S R}=\kappa_{R D_{f}} \neq 0\right)$

$$
\begin{aligned}
\mathrm{P}_{\text {out }}^{D_{f}, n i} & =1-\frac{2}{\Gamma\left(\alpha_{S R}\right) \beta_{S R}^{\alpha_{S R}}} e^{-\frac{\lambda}{\beta_{S R}}-\frac{b_{1} \lambda}{b_{2} \beta_{R D_{f}}}} \sum_{g_{R D_{f}}=0}^{\alpha_{\text {RD }}-1} \sum_{j=0}^{-1} \sum_{S=0}^{\alpha_{S R}-1} \sum_{R D_{f}}\left(\begin{array}{c}
\alpha_{S R}-1 \\
j
\end{array}\right)\left(\begin{array}{c}
g_{R D_{f}} \\
n
\end{array}\right)\left(\frac{\lambda}{b_{2} \beta_{R D_{f}}}\right)^{g_{R D_{f}}} \\
& \times \frac{\lambda_{S R}^{\alpha_{S R}-1-j}}{g_{R D_{f}} !}\left(b_{1} \lambda+c\right)^{n}\left(b_{1}\right)^{g_{R D_{f}}-n}\left[\frac{\lambda \beta_{S R}\left(b_{1} \lambda+c\right)}{b_{2} \beta_{R D_{f}}}\right]^{\frac{j-n+1}{2}} K_{j-n+1}\left[2 \sqrt{\frac{\lambda\left(b_{1} \lambda+c\right)}{b_{2} \beta_{S R} \beta_{R D_{f}}}}\right]
\end{aligned}
$$

where $\lambda=b_{2} c \gamma_{t h f} /\left(a_{f}-a_{n} \gamma_{t h f}-d_{1} \gamma_{t h f}\right)$ with $\gamma_{t h f}\left(a_{n}+d_{1}\right)<a_{f}$, and $c=1 / \gamma . K_{v}(\cdot)$ denotes the vth-order modified Bessel function of the second kind as defined in [23].

Proof: See Appendix A.

- Ideal condition $\left(\kappa_{S R}=\kappa_{R D_{f}}=0\right)$

$$
\begin{aligned}
\mathrm{P}_{\text {out }}^{D_{f}, \text { id }}=1-\frac{2}{\Gamma\left(\alpha_{S R}\right) \beta_{S R}^{\alpha_{S R}}} e^{-\frac{\lambda}{\beta_{S R}}-\frac{\lambda}{\beta_{R D_{f}}}} \sum_{g_{R D_{f}}=0}^{\alpha_{R D_{f}}-1} \sum_{j=0}^{\alpha_{S R}-1} \sum_{n=0}^{g_{R D_{f}}}\left(\begin{array}{c}
\alpha_{S R}-1 \\
j
\end{array}\right)\left(\begin{array}{c}
g_{R D_{f}} \\
n
\end{array}\right)\left(\frac{\lambda}{\beta_{R D_{f}}}\right)^{g_{R D_{f}}} \\
\times \frac{\lambda_{S R^{\prime}-1-j}^{\alpha_{1}}}{g_{R D_{f}} !}(\lambda+c)^{n}\left[\frac{\lambda \beta_{S R}(\lambda+c)}{\beta_{R D_{f}}}\right]^{\frac{j-n+1}{2}} K_{j-n+1}\left[2 \sqrt{\frac{\lambda(\lambda+c)}{\beta_{S R} \beta_{R D_{f}}}}\right]
\end{aligned}
$$

where $\lambda=c \gamma_{t h f} /\left(a_{f}-a_{n} \gamma_{t h f}\right)$ with a necessary condition $a_{n} \gamma_{t h f}<a_{f}$. If the condition is not satisfied, the user $D_{f}$ cannot decode its signal successfully irrespective of the channel SNR.

Proof: Follows trivially by substituting (11) into (14) and letting $\kappa_{S R}=\kappa_{R D_{f}}=0$.

Remark 1: As seen from Theorem 1, we observe that owing to the impact of hardware impairments, the outage probability of (15) is determined by the distortion noise and fading parameters, while result of (16) is only determined by the fading parameters. In addition, the outage performances of (15) and (16) are limited by the relay.

2) Outage Probability for $D_{n}$

At $D_{n}$, the received signals are processed by using SC algorithm, which the maximal SINR is selected between $\tilde{\gamma}_{R D_{n}}$ and $\tilde{\gamma}_{S D_{n}}$. The outage event will occur for $D_{n}$ if $D_{n}$ cannot decode $D_{f}$ 's signal or also cannot decode its own signal during the two time slots. Therefore, the 
outage probability of $D_{n}$ can be expressed as follows:

$$
\mathrm{P}_{\text {out }}^{D_{n}}=\underbrace{\left[1-\operatorname{Pr}\left(\tilde{\gamma}_{S D_{f \rightarrow n}} \geq \gamma_{t h f}, \tilde{\gamma}_{S D_{n}} \geq \gamma_{t h n}\right)\right]}_{\mathrm{I}_{2}} \underbrace{\left[1-\operatorname{Pr}\left(\tilde{\gamma}_{R D_{f \rightarrow n}} \geq \gamma_{t h f}, \tilde{\gamma}_{R D_{n}} \geq \gamma_{t h n}\right)\right]}_{\mathrm{I}_{3}}
$$

where $\gamma_{t h n}$ denotes the outage threshold at $D_{n}$.

- Non-ideal condition $\left(\kappa_{S R}=\kappa_{R D_{f}}=\kappa_{R D_{n}}=\kappa_{S D_{n}} \neq 0\right)$

The following theorem provides new closed-form expressions for $D_{n}$ in the presence of non-ideal hardware impairments.

Theorem 2: For Nakagami- $m$ fading channels, the exact closed-form expression for the outage probability of $D_{n}$ in presence of hardware impairments is given as

$$
\begin{aligned}
\mathrm{P}_{\text {out }}^{D_{n}, n i}=\left[1-\sum_{g_{S S_{n}}=0}^{\alpha_{S D_{n}}-1} \frac{1}{g_{S D_{n}} !} e^{-\frac{\tau}{\beta_{S D_{n}}}}\left(\frac{\tau}{\beta_{S D_{n}}}\right)^{g_{S D_{n}}}\right]\left\{1-\frac{2}{\Gamma\left(\alpha_{S R}\right) \beta_{S R}^{\alpha_{S R}}} e^{-\frac{\theta}{\beta_{S R}}-\frac{b_{1} \theta}{b_{3} \beta_{R D_{n}}}}\right. \\
\quad \times \sum_{g_{R D_{n}}=0}^{\alpha_{R D_{n}}-1} \sum_{j=0}^{-\alpha_{S R}-1 g_{R D_{n}}} \sum_{n=0}^{\alpha_{S R}}\left(\begin{array}{c}
\alpha_{S R}-1 \\
j
\end{array}\right)\left(\begin{array}{c}
g_{R D_{n}} \\
n
\end{array}\right)\left(\frac{\theta}{b_{3} \beta_{R D_{n}}}\right)^{g_{R D_{n}}} \frac{\theta^{\alpha_{S R}-1-j}}{g_{R D_{n}} !}\left(b_{1} \theta+c\right)^{n} \\
\left.\quad \times\left(b_{1}\right)^{g_{R D_{n}}-n}\left[\frac{\theta \beta_{S R}\left(b_{1} \theta+c\right)}{b_{3} \beta_{R D_{n}}}\right]^{\frac{j-n+1}{2}} \mathrm{~K}_{j-n+1}\left[2 \sqrt{\frac{\theta\left(b_{1} \theta+c\right)}{b_{3} \beta_{S R} \beta_{R D_{n}}}}\right]\right\}
\end{aligned}
$$

where $\tau=\max \left(\tau_{1}, \tau_{2}\right), \tau_{1}=c \gamma_{\text {thf }} /\left(a_{f}-\left(a_{n}+\kappa_{S D_{n}}^{2}\right) \gamma_{\text {thf }}\right), \tau_{2}=c \gamma_{\text {thn }} /\left(a_{n}-\kappa_{S D_{n}}^{2} \gamma_{\text {thn }}\right) ; \theta=\max \left(\theta_{1}, \theta_{2}\right), \theta_{1}=b_{3} c \gamma_{\text {thf }} /\left(a_{1}-\left(a_{2}-d_{2}\right) \gamma_{\text {thf }}\right)$, $\theta_{2}=b_{3} c \gamma_{t h n} /\left(a_{2}-d_{2} \gamma_{t h n}\right)$. Note that (18) is obtained by assuming the following condition holds $\gamma_{t h f}\left(a_{n}+\kappa_{S D_{n}}^{2}\right)>a_{f}, \gamma_{t h n} \kappa_{S D_{n}}^{2}>a_{n}$.

Proof: See Appendix B.

- Ideal condition $\left(\kappa_{S R}=\kappa_{R D_{f}}=\kappa_{R D_{n}}=\kappa_{S D_{n}}=0\right)$

The following theorem provides new closed-form expression for $D_{n}$ in the presence of ideal hardware.

$$
\begin{aligned}
\mathrm{P}_{\text {out }}^{D_{n}, i d}=[1 & \left.-\sum_{g_{S D_{n}}=0}^{\alpha_{S D_{n}}-1} \frac{1}{g_{S D_{n}} !} e^{-\frac{\tau}{\beta_{S D_{n}}}}\left(\frac{\tau}{\beta_{S D_{n}}}\right)^{g_{S D_{n}}}\right]\left\{1-\frac{2}{\Gamma\left(\alpha_{S R}\right) \beta_{S R}^{\alpha_{S R}}} e^{-\frac{\theta}{\beta_{S R}}-\frac{\theta}{\beta_{R D_{n}}}} \sum_{g_{R D_{n}}=0}^{\alpha_{R D_{n}}-1} \sum_{j=0} \sum_{S R=0}-1 g_{R D_{n}}\left(\begin{array}{c}
\alpha_{S R}-1 \\
j
\end{array}\right)\right. \\
& \left.\times\left(\begin{array}{c}
g_{R D_{n}} \\
n
\end{array}\right)\left(\frac{\theta}{\beta_{R D_{n}}}\right)^{g_{R D_{n}}} \frac{\theta^{\alpha_{S R}-1-j}}{g_{R D_{n}} !}(\theta+c)^{n}\left[\frac{\theta \beta_{S R}(\theta+c)}{\beta_{R D_{n}}}\right]^{\frac{j-n+1}{2}} \mathrm{~K}_{j-n+1}\left[2 \sqrt{\frac{\theta(\theta+c)}{\beta_{S R} \beta_{R D_{n}}}}\right]\right\}
\end{aligned}
$$

where $\tau=\max \left(\tau_{1}, \tau_{2}\right), \tau_{1}=c \gamma_{\text {thf }} /\left(a_{f}-a_{n} \gamma_{\text {thf }}\right), \tau_{2}=c \gamma_{\text {thn }} / a_{n} ; \theta=\max \left(\theta_{1}, \theta_{2}\right), \theta_{1}=c \gamma_{\text {th }} /\left(a_{1}-a_{2} \gamma_{\text {thf }}\right), \theta_{2}=c \gamma_{\text {thn }} / a_{2}$. Note that (19) is obtained by assuming the following condition holds $a_{n} \gamma_{t h f}>a_{f}$.

Proof: Follows trivially by substituting (7), (8), (12) and (13) into (17) and letting $\kappa_{S R}=\kappa_{R D_{f}}=\kappa_{R D_{n}}=\kappa_{S D_{n}}=0$.

Remark 2: Although the results of Theorem 1 and Theorem 2 can be expressed in closed-form and can be efficiently evaluated, they do not offer the insights of fading parameters and distortion noise on the outage probability. In the following, the asymptotic outage behaviors for $D_{f}$ and $D_{n}$ are examined.

\subsection{Asymptotic Outage Behavior}

In order to gain more insights, we focus on the asymptotic analysis for the outage probability at high SNR regime by characterizing the CDF of the channel gains in the high SNR regime. 
For high SNR regime, the asymptotic CDF of the channel gain $\rho_{i}$ can be given by [14]

$$
F_{\rho_{i}}^{\infty}(x) \approx \frac{1}{\alpha_{i} !}\left(\frac{x}{\beta_{i}}\right)^{\alpha_{i}}
$$

Based on (6), the CDF $F_{\rho_{i}}(x)$ can be approximated dominant term $F_{\rho_{i}}^{\infty}(x)$. Therefore, the asymptotic outage probability for $D_{f}$ and $D_{n}$ are presented in Corollary 1 and Corollary 2, respectively.

- Non-ideal condition $\left(\kappa_{S R}=\kappa_{R D_{f}}=\kappa_{R D_{n}}=\kappa_{S D_{n}} \neq 0\right)$

Corollary 1: For Nakagami- $m$ fading channels, the asymptotic closed-form for the outage probability of $D_{f}$ is given as

$$
\begin{aligned}
\mathrm{P}_{D_{f}, n i}^{\infty, i} \approx \frac{1}{\alpha_{S R} !}\left(\frac{\lambda}{\beta_{S R}}\right)^{\alpha_{S R}} & +\frac{1}{\Gamma\left(\alpha_{S R}\right) \beta_{S R}^{\alpha_{S R}} \alpha_{R D_{f}} !} \sum_{j=0}^{\alpha_{R D_{f}}}\left(\begin{array}{c}
\alpha_{R D_{f}} \\
j
\end{array}\right)\left(\frac{\lambda}{b_{2} \beta_{R D_{f}}}\right)^{\alpha_{R D_{f}}} \\
& \times\left(\alpha_{S R}-1-j\right) !\left(b_{1} \lambda+c\right)^{j}\left(\beta_{S R}\right)^{\alpha_{S R}-j}\left(b_{1}\right)^{\alpha_{R D_{f}}-j}
\end{aligned}
$$

Proof: See Appendix C.

Corollary 2: For Nakagami-m fading channels, the asymptotic closed-form for the outage probability of $D_{n}$ is obtained as

$$
\begin{array}{r}
\mathrm{P}_{D_{n}, n i}^{\infty, n i} \frac{1}{\Gamma\left(\alpha_{S R}\right) \beta_{S R}^{\alpha_{S R}} \alpha_{S D_{n}} ! \alpha_{R D_{n}} !} \sum_{j=0}^{\alpha_{R D_{n}}}\left(\begin{array}{c}
\alpha_{R D_{n}} \\
j
\end{array}\right)\left(\frac{\theta}{b_{3} \beta_{R D_{n}}}\right)^{\alpha_{R D_{n}}}\left(\frac{\tau}{\beta_{S D_{n}}}\right)^{\alpha_{S D_{n}}} \\
\times\left(\alpha_{S R}-1-j\right) !\left(b_{1} \theta+c\right)^{j}\left(\beta_{S R}\right)^{\alpha_{S R}-j}\left(b_{1}\right)^{\alpha_{R D_{f}}-j}
\end{array}
$$

Proof: See Appendix D.

- Ideal condition $\left(\kappa_{S R}=\kappa_{R D_{f}}=\kappa_{R D_{n}}=\kappa_{S D_{n}}=0\right)$

The following theorem provides the asymptotic outage probabilities for $D_{f}$ and $D_{n}$ in the presence of ideal hardware.

$$
\begin{aligned}
& \mathrm{P}_{D_{f}, \text { id }}^{\infty} \approx \frac{1}{\alpha_{S R} !}\left(\frac{\lambda}{\beta_{S R}}\right)^{\alpha_{S R}}+\frac{\left(\alpha_{S R}-1-j\right) !}{\Gamma\left(\alpha_{S R}\right) \beta_{S R}^{\alpha_{S R}} \alpha_{R D_{f}} !} \sum_{j=0}^{\alpha_{R f_{f}}}\left(\begin{array}{c}
\alpha_{R D_{f}} \\
j
\end{array}\right)\left(\frac{\lambda}{\beta_{R D_{f}}}\right)^{\alpha_{R D_{f}}}(\lambda+c)^{j}\left(\beta_{S R}\right)^{\alpha_{S R}-j} \\
& \mathrm{P}_{D_{n}}^{\infty, i d} \approx \frac{\left(\alpha_{S R}-1-j\right) !}{\Gamma\left(\alpha_{S R}\right) \beta_{S R}^{\alpha_{S R}} \alpha_{S D_{n}} ! \alpha_{R D_{n}} !} \sum_{j=0}^{\alpha_{R D_{n}}}\left(\begin{array}{c}
\alpha_{R D_{n}} \\
j
\end{array}\right)\left(\frac{\theta}{\beta_{R D_{n}}}\right)^{\alpha_{R D_{n}}}\left(\frac{\tau}{\beta_{S D_{n}}}\right)^{\alpha_{S D_{n}}}(\theta+c)^{j}\left(\beta_{S R}\right)^{\alpha_{S R}-j}
\end{aligned}
$$

Proof: The proof follows trivially by taking $\kappa_{S R}=\kappa_{R D_{f}}=\kappa_{R D_{n}}=\kappa_{S D_{n}}=0$ in (21) and (22), we can obtain (23) and (24) after some simplifications.

Remark 3: From (22)-(24), we can observe that at high SNR regime, the effect of fading parameters on the outage probability can be decomposed. In addition, the above results indicate that the outage probability of the user $D_{f}$ only depends on the fading parameters of relay link $\left(\alpha_{S R}, \alpha_{R D_{f}}, \beta_{S R}, \beta_{R D_{f}}\right)$, while the outage probability of user $D_{n}$ is limited by the fading parameters of both direct and relay links $\left(\alpha_{S R}, \alpha_{R D_{f}}, \alpha_{S D_{n}}, \beta_{S R}, \beta_{R D_{f}}, \beta_{S D_{n}}\right)$.

\section{The Ergodic Sum Rate of NOMA}

In this section, the ergodic sum rates of NOMA relaying networks over Nakagami-m fading channels with ideal hardware and hardware impairments are investigated. 


\subsection{Approximate Analysis}

We consider that the target SINRs of two users are determined by the users' channel condition. In this case, $\tilde{\gamma}_{R D_{t \rightarrow n}} \geq \tilde{\gamma}_{R D_{n}}$ may not be correct when $\rho_{R D_{n}}<\rho_{R D_{f}}$ [24]. Therefore, the achievable rate of $D_{f}$ and $D_{n}$ can be written as

$$
\begin{gathered}
R_{D_{f}}=\frac{1}{2} \log _{2}\left(1+\tilde{\gamma}_{R D_{f}}\right) \\
R_{D_{n}}=\left\{\begin{array}{l}
\frac{1}{2} \log _{2}\left(1+\max \left[\tilde{\gamma}_{S D_{n}}, \tilde{\gamma}_{R D_{n}}\right]\right), \text { if } \rho_{R D_{n}}>\rho_{R D_{f}} \\
\frac{1}{2} \log _{2}\left(1+\tilde{\gamma}_{S D_{n}}\right), \text { if } \rho_{R D_{n}}<\rho_{R D_{f}}
\end{array}\right.
\end{gathered}
$$

The ergodic sum rate of the two users can be expressed as

$$
\begin{aligned}
& R_{\text {ave }}^{D_{f}}=\frac{1}{2} \mathrm{E}\left[\log _{2}\left(1+\tilde{\gamma}_{R D_{f}}\right)\right] \\
& R_{a v e}^{D_{n}}=\operatorname{Pr}\left(\rho_{R D_{n}}>\rho_{R D_{f}}\right) \underbrace{\mathrm{E}\left[\frac{1}{2} \log _{2}\left(1+\max \left[\tilde{\gamma}_{S D_{n}}, \tilde{\gamma}_{R D_{n}}\right]\right)\right]}_{\mathrm{P}^{D_{n}, 1}} \\
& +\operatorname{Pr}\left(\rho_{R D_{n}}<\rho_{R D_{f}}\right) \underbrace{\mathrm{E}\left[\frac{1}{2} \log _{2}\left(1+\tilde{\gamma}_{S D_{n}}\right)\right]}_{R_{n \in D_{n}, 2}^{D_{n}}}
\end{aligned}
$$

By applying the approximation $\mathrm{E}\{\log 2(1+x / y)\} \approx \log 2(1+\mathrm{E}\{x\} / \mathrm{E}\{y\})$, the ergodic sum rate over Nakagami- $m$ fading channels by extracting out the average fading power as $\mathrm{E}_{\rho_{i}}\left\{\rho_{i}\right\}=\alpha_{i} \beta_{i}$ is provided in the following theorem.

Theorem 3: Considering $\rho_{R D_{f}}$ and $\rho_{R D_{n}}$ independent identically distributed random variables, we have $\operatorname{Pr}\left(\rho_{R D_{f}}>\rho_{R D_{n}}\right)=\operatorname{Pr}\left(\rho_{R D_{f}}<\rho_{R D_{n}}\right)=1 / 2$. Therefore, for Nakagami- $m$ fading channels, the approximate closed-form expression for the ergodic sum rate of NOMA relaying network in the presence of hardware impairments is expressed as

- Non-ideal condition $\left(\kappa_{S R}=\kappa_{R D_{f}}=\kappa_{R D_{n}}=\kappa_{S D_{n}} \neq 0\right)$

$$
\begin{aligned}
R_{\text {ave }}^{\text {sum } n \text { i }} & \approx \\
& \frac{1}{4}\left[2 \log _{2}\left(1+\frac{a_{f} \gamma^{2} \hat{\rho}_{S R} \hat{\rho}_{R D_{f}}}{\left(a_{n}+d_{1}\right) \gamma^{2} \hat{\rho}_{S R} \hat{\rho}_{R D_{f}}+b_{1} \gamma \hat{\rho}_{S R}+b_{2} \gamma \hat{\gamma}_{R D_{f}}+1}\right)\right. \\
& +\log _{2}\left(1+\max \left[\frac{a_{n} \gamma \hat{\rho}_{S D_{n}}}{\kappa_{S D_{n}}^{2} \gamma \hat{\rho}_{S D_{n}}+1}, \frac{a_{n} \gamma^{2} \hat{\rho}_{S R} \hat{\rho}_{R D_{n}}}{d_{2} \gamma^{2} \hat{\rho}_{S R} \hat{\rho}_{R D_{n}}+b_{1} \gamma \hat{\rho}_{S R}+b_{3} \gamma \hat{\rho}_{R D_{n}}+1}\right]\right) \\
& \left.+\log _{2}\left(1+\frac{a_{n} \gamma \hat{\rho}_{S D_{n}}}{\kappa_{S D_{n}}^{2} \gamma \hat{\rho}_{S D_{n}}+1}\right)\right]
\end{aligned}
$$

where $\hat{\rho}_{i}=\alpha_{i} \beta_{i}$ for $i \in\left\{S R, R D_{f}, R D_{n}, S D_{n}\right\}$.

- Ideal condition $\left(\kappa_{S R}=\kappa_{R D_{f}}=\kappa_{R D_{n}}=\kappa_{S D_{n}}=0\right)$

$$
\begin{aligned}
& R_{\text {ave }}^{\text {sum } i d} \approx \frac{1}{4}\left[2 \log _{2}\left(1+\frac{a_{f} \gamma^{2} \hat{\rho}_{S R} \hat{\rho}_{R D_{f}}}{a_{n} \gamma^{2} \hat{\rho}_{S R} \hat{\rho}_{R D_{f}}+\gamma \hat{\rho}_{S R}+\gamma \hat{\rho}_{R D_{f}}+1}\right)\right. \\
& \left.+\log _{2}\left(1+\max \left[a_{n} \gamma \hat{\rho}_{S D_{n}}, \frac{a_{n} \gamma^{2} \hat{\rho}_{S R} \hat{\rho}_{R D_{n}}}{\gamma \hat{\rho}_{S R}+\gamma \hat{\rho}_{R D_{n}}+1}\right]\right)+\log _{2}\left(1+a_{n} \gamma \hat{\rho}_{S D_{n}}\right)\right]
\end{aligned}
$$

Remark 4: Although the result of Theorem 3 can be expressed in closed-form and can be efficiently evaluated, it does not offer the insights of fading parameters and distortion noise on 
the sum rate. In the following, the asymptotic ergodic sum rate for NOMA DH AF relaying networks in presence of hardware impairments is examined. For comparison, the upper bound with ideal conditions is also analysed.

\subsection{Asymptotic and Upper Bound Analyses}

In this subsection, we focus on the asymptotic analysis in terms of the ergodic sum rate with hardware impairments and the upper bound for ergodic sum rate of ideal hardware as follows.

Corollary 3: Under the assumption of i.i.d. Nakagami- $m$ channel, when $\gamma \rightarrow \infty$, the ergodic sum rate of NOMA DH AF relaying networks can be obtained as

- Non-ideal condition $\left(\kappa_{S R}=\kappa_{R D_{f}}=\kappa_{R D_{n}}=\kappa_{S D_{n}} \neq 0\right)$

$$
R_{\text {ave }}^{\text {sum } n i} \approx \frac{1}{2} \log _{2}\left(1+\frac{a_{f}}{a_{n}+d_{1}}\right)+\frac{1}{4} \log _{2}\left(1+\frac{a_{n}}{\hat{k}}\right)+\frac{1}{4} \log _{2}\left(1+\frac{a_{n}}{\kappa_{S D_{n}}^{2}}\right)
$$

where $\hat{k}=\min \left(\kappa_{S D_{n}}^{2}, d_{2}\right)$.

Proof: See Appendix E.

From Corollary 3, we can acquire that $R_{\text {ave }}^{\text {sum ni }}$ is a constant and independent of the channel condition. In addition, the value of $R_{\text {ave }}^{\text {sum }}$ ni depends on the power allocation coefficients and the level of impairments in the high SNR regime.

In the case of ideal hardware, it is difficult to obtain the asymptotic expression for the ergodic sum rate of ideal hardware due to the high complexity of integrals. Therefore, an upper bound for the ergodic sum rate is given in the following corollary.

- Ideal condition $\left(\kappa_{S R}=\kappa_{R D_{f}}=\kappa_{R D_{n}}=\kappa_{S D_{n}}=0\right)$

Corollary 4: In the case of ideal hardware, when $\gamma \rightarrow \propto$ with a finite non-zero ratio, the upper bound for the ergodic sum rate will be

$$
\begin{aligned}
& R_{\text {ave }}^{\text {sum } i d} \leq \frac{1}{2} \log _{2}\left(1+\frac{a_{f}}{a_{n}}\right)+\frac{1}{2 \ln 2} \sum_{g_{S D_{n}}}^{\alpha_{S D_{n}}-1} \frac{1}{g_{S D_{n}} !}\left(f_{1}\right)^{g_{S D_{n}}} \Phi_{1}+\frac{1}{4 \ln 2} \\
& \times\left[\sum_{g_{S R}=0}^{\alpha_{S R}-1} \sum_{g_{R D_{n}}=0}^{\alpha_{R_{D_{n}}}-1} \frac{1}{g_{S R} ! g_{R D_{n}} !}\left(f_{2}\right)^{g_{S R}}\left(f_{3}\right)^{g_{R D_{n}}} \Phi_{2} \sum_{g_{S R}=0}^{\alpha_{S R}-1} \sum_{g_{R D_{n}}=0}^{\alpha_{R D_{n}}-1}\right. \\
& \left.\times \sum_{g_{S D_{n}}=0}^{\alpha_{S D_{n}}-1} \frac{1}{g_{S R} ! g_{R D_{n}} ! g_{S D_{n}} !}\left(f_{1}\right)^{g_{S D_{n}}}\left(f_{2}\right)^{g_{S R}}\left(f_{3}\right)^{g_{R D_{n}}} \Phi_{3}\right]
\end{aligned}
$$

Proof: See Appendix F.

Remark 5: For non-ideal conditions, there is a rate ceiling for the ergodic sum rate, which is irrelated to the average transmit SNR. It means that it is not always beneficial to the ergodic sum rate by increasing the average transmit SNR. For ideal conditions, the ergodic sum rate increases when the average grows into infinity.

\section{Numerical Results}

In this section, the correctness of the theoretical analytical results is verified by a set of numerical simulations. Meanwhile, the performance of NOMA relaying networks with ideal hardware is also provided. Unless otherwise specified, we set the power allocation coefficients $a_{f}=3 / 4, a_{n}=1 / 4, \gamma_{t h f}=1, \gamma_{t h n}=3$. In addition, we consider the variance of AWGN $N_{0}=1$, and the design parameters $\kappa_{S R}=\kappa_{R D_{f}}=\kappa_{R D_{n}}=\kappa_{S D_{n}}=\kappa$. 


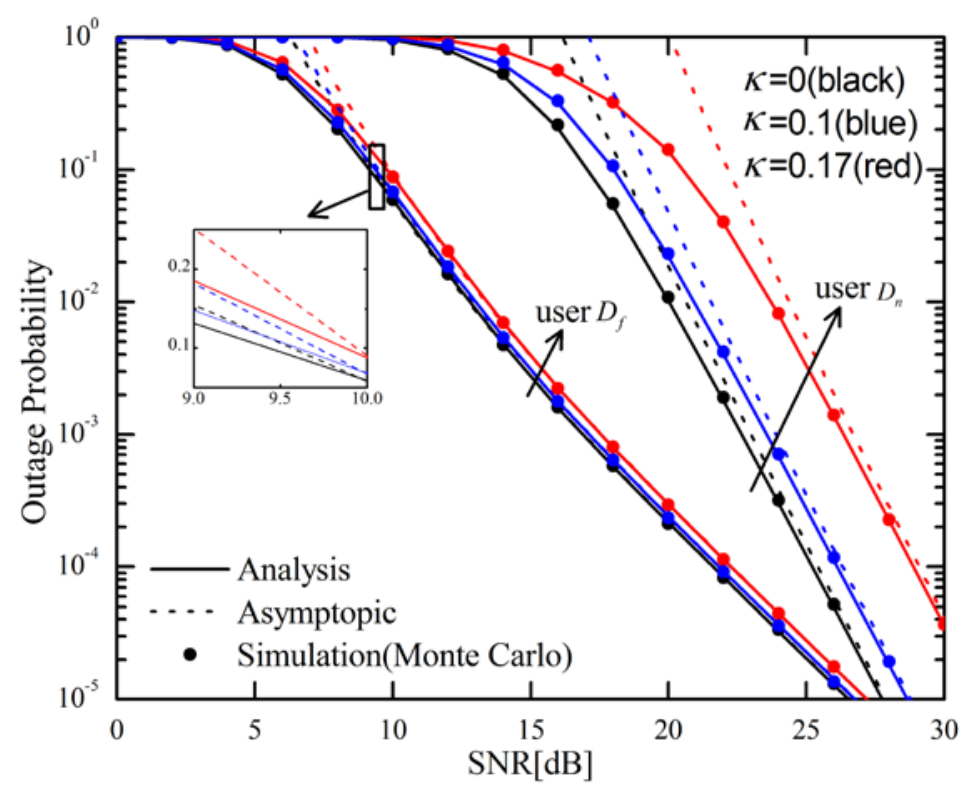

Fig. 2. Outage probability against transmit SNR

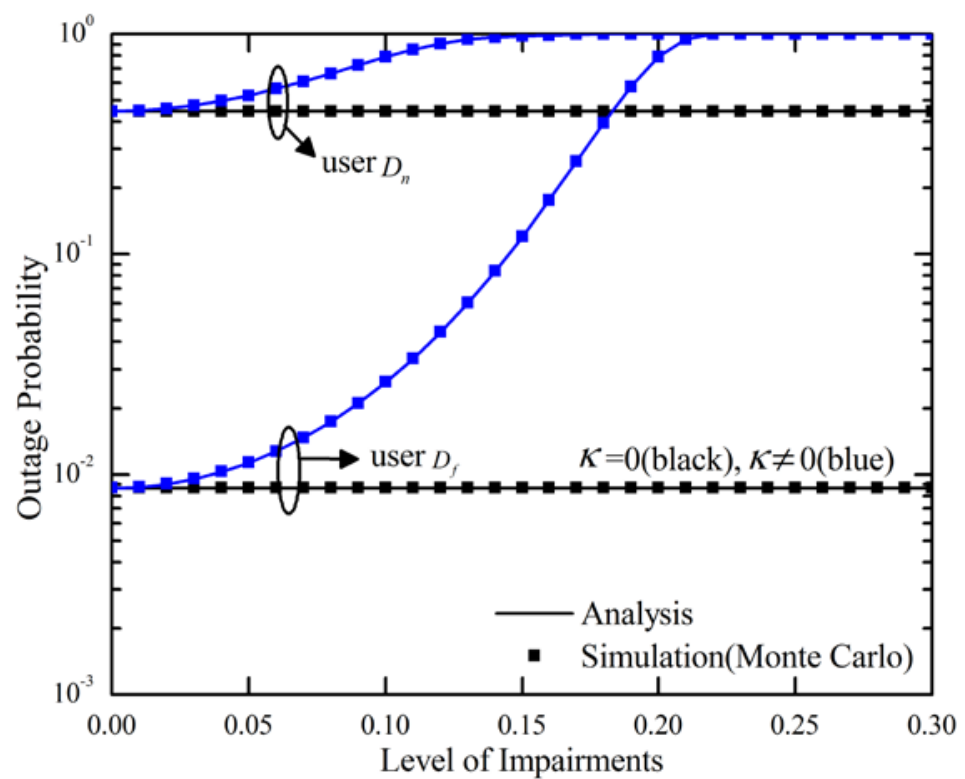

Fig. 3. Outage probability against the level of impairments

Fig. 2 shows the outage probability of NOMA relaying networks versus the transmit SNR with different levels of impairments $\kappa \in\{0,0.1,0.17\}$, where the value of fading parameters are set as: $\alpha_{S R}=4, \alpha_{R D_{f}}=\alpha_{R D_{n}}=\alpha_{S D_{n}}=2 ; \beta_{S R}=\beta_{R D_{n}}=\beta_{S D_{n}}=1, \beta_{R D_{f}}=4$. In addition, we set the transmit power $\mathrm{P}_{S}=\mathrm{P}_{R}=\mathrm{P}$. It is noticed that the curves of analytical expressions in (15), (16), (18) and (19) are perfect agreement with Monte-Carlo simulation results, which demonstrate the correctness of our analyses in Section 3. Furthermore, we compare the asymptotic outage probability of (21)-(24) with the analytical outage probability for different level of impairments. For $D_{f}$, we can observe that the curves of ideal analytical outage probability and impaired analytical outage probability overlap with the asymptotic outage probability when 
SNR $>10 \mathrm{~dB}$. For $D_{n}$, we can observe that the outage probability gap between ideal hardware and hardware impairments curves becomes larger as average SNR get larger. All these reasons are that hardware impairments can degrade the performance of communication systems. In addition, it can be readily noticed from Fig. 2 that the asymptotic outage probability curve is sufficiently tight across the analytical expressions in the high SNR. When the aggregate level of hardware impairments $\kappa$ becomes large, the gap between ideal analytical and impaired analytical outage probability curves becomes large for the same average SNR due to the large distortion noise.

Fig. 3 shows the impact of hardware impairments on the outage probability for NOMA DH AF relaying network when $\alpha_{S R}=\alpha_{S D_{n}}=7, \alpha_{R D_{f}}=6, \alpha_{R D_{n}}=3 ; \beta_{S R}=3, \beta_{S D_{n}}=1, \beta_{R D_{f}}=\beta_{R D_{n}}=7$, and the transmit SNR=15. In addition, the outage threshold at $D_{f}$ and $D_{n}$ are denoted by $\gamma_{\text {thf }}=2$ and $\gamma_{t h n}=3.5$. A specific observation is that the performance of outage probability decreases as the level of impairments increasing. When $\kappa$ is larger than 0.2 , the outage probability of the two users are zeros due the severe hardware impairments. Fig. 3 also indicates that the outage probability of the user $D_{f}$ is better than the user $D_{n}$ in the low level of the impairments due to the large power allocation factor.

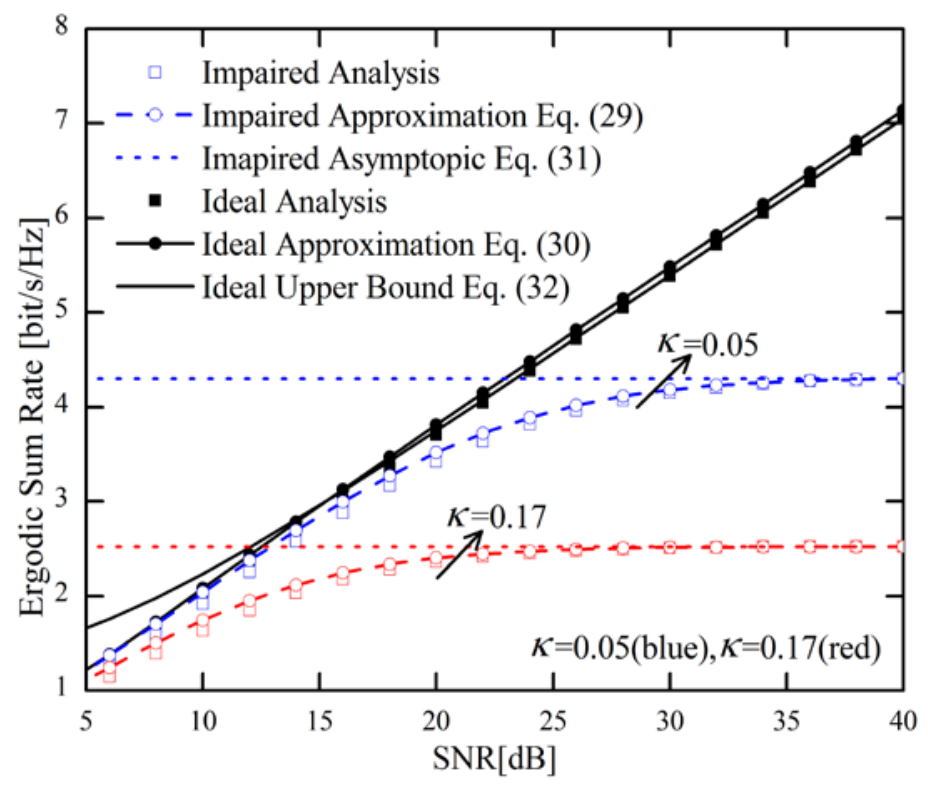

Fig. 4. Ergodic sum rate against transmit SNR

Fig. 4 plots the ergodic sum rate of the NOMA DH AF relaying networks with ideal hardware and hardware impairments where the values of parameters are set as: $\alpha_{S R}=\alpha_{R D_{n}}=2, \alpha_{R D_{f}}=7, \alpha_{S D_{n}}=4 ; \beta_{S R}=\beta_{R D_{f}}=\beta_{S D_{n}}=7, \beta_{R D_{n}}=4$ and $\kappa=\{0,0.05,0.17\}$.

From Fig. 4, we note that hardware impairments have a small impact on the sum rate at low SNRs, but have a significant difference at high SNRs. In addition, we note that for non-ideal conditions, there are rate ceilings for the sum rate, which are irrelated to the average SNR. For ideal conditions, the sum rates grow logarithmically with the average SNR. The upper bound of the sum rate is shown to be tightly bound in the high SNR regime. Moreover, for the same SNR, as the coefficient of hardware impairments becomes smaller, the ergodic sum rate achieves large. 


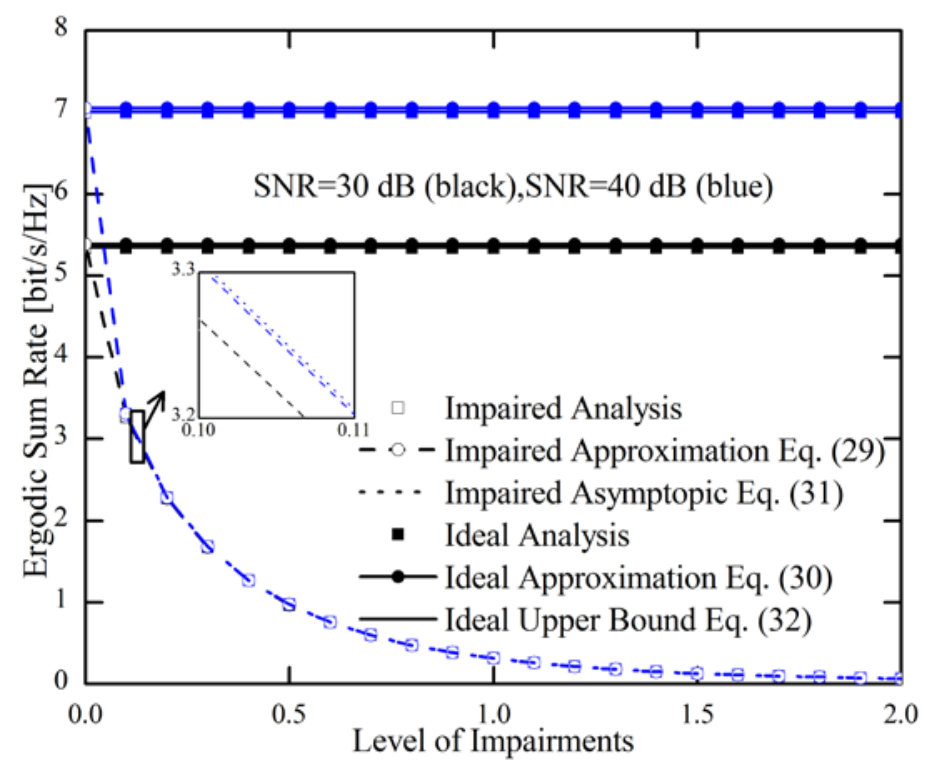

Fig. 5. Ergodic sum rate against the level of impairments

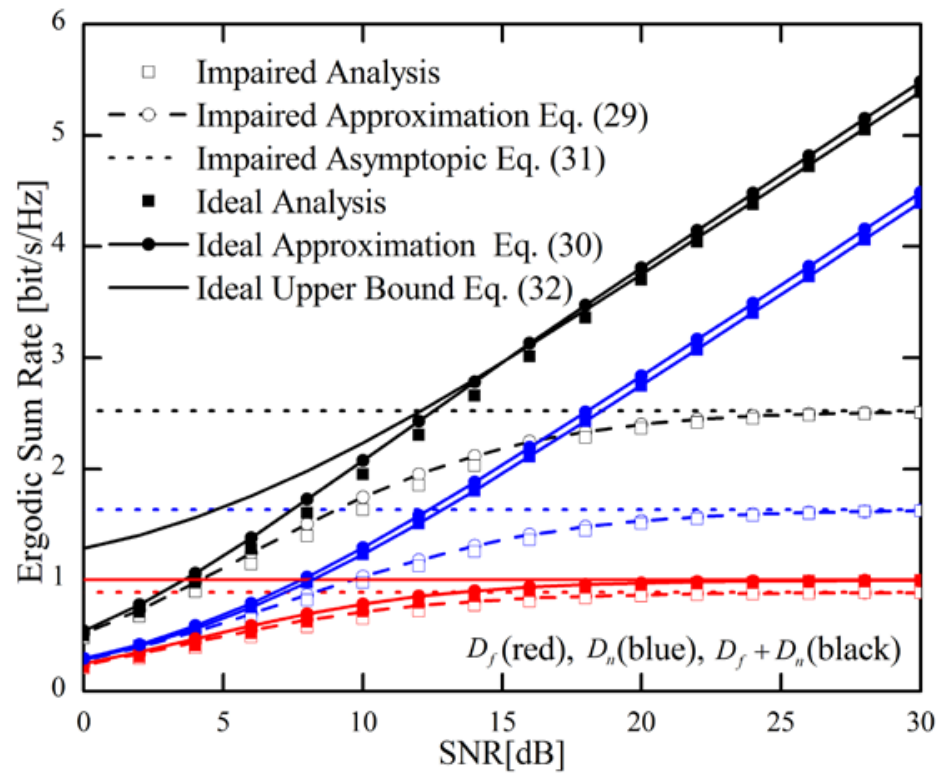

Fig. 6. Ergodic sum rate against transmit SNR

Fig. 5 investigates the impact of hardware impairments on the ergodic sum rate for NOMA DH AF relaying network with different average $\operatorname{SNR} \in\{30,40\}$. For comparison, the ideal hardware impacts to the ergodic sum rate are also taken into consideration. In this simulation, we set $\alpha_{S R}=\alpha_{R D_{n}}=2, \alpha_{R D_{f}}=\alpha_{S D_{n}}=7 ; \beta_{S R}=\beta_{R D_{n}}=2, \beta_{R D_{f}}=7, \beta_{S D_{n}}=1$. We can observe that the curves of impaired rate ceiling for ergodic sum rate between the average $\mathrm{SNR}=30 \mathrm{~dB}$ and $\mathrm{SNR}=40 \mathrm{~dB}$ are almost overlapped for all values of $\kappa$ except $\kappa=0$ which is irrelevant to the SNR. In addition, the ergodic sum rate of ideal hardware is a fixed value, and the impaired ergodic sum rate approaches to zero when the level impairments get larger due to the severe hardware 
impairments. The figure also points out that the impaired ergodic sum rate decrease rapidly when the level of impairments is below 0.5 .

Fig. 6 shows the ergodic sum rate of the NOMA DH AF relaying networks versus the average SNR. In this simulation, we set $\alpha_{S R}=\alpha_{R D_{n}}=2, \alpha_{R D_{f}}=7, \alpha_{S D_{n}}=4 ; \beta_{S R}=\beta_{R D_{f}}=\beta_{S D_{n}}=7$, $\beta_{R D_{n}}=4$ and $\kappa=0.17$. For comparison, the performance with ideal conditions is also taken into account. More precisely, It is noticed from Fig.6 that the ideal sum rate of $D_{f}$ approaches to a constant value in the high SNR regime which is saturating and approaching $\frac{1}{2} \log _{2}\left(1+\frac{a_{f}}{a_{n}}\right)$, as proved by Corollary 4 . The ideal sum rate of user $D_{n}$ increases linearly with the transmit SNR. Finally, we can also observe that there are rate ceilings for the sum rate of user $D_{f}$ and $D_{n}$ due to the hardware impairments, which means that add the average SNR is not always beneficial to the system performance.

\section{Conclusion}

In this paper, the performance of relaying networks using NOMA under the condition that ideal and impaired hardware were investigated. The exact closed-form expressions for the outage probability and ergodic sum rate were derived. Based on the analytical results, the upper bound for the outage probability have been obtained. Additionly, the asymptotic analyses at high SNR for the outage probability and ergodic sum rate are examined. It is observed that the ergodic sum rate degraded in NOMA relaying networks due to the exist of hardware impairments. Moreover, the performance of these two users was compared in terms of outage performance and ergodic sum rate, it is noteworthy that the outage probability of the user without the direct link to the source is better than the user with a direct link to the source in the low SNR due to the allocated power, which means that NOMA can improve the fairness among serviced users.

\section{APPENDIX A PROOF OF THEOREM 1}

Substituting (11) into (14), the outage probability of $D_{f}$ turns out to be

$$
\begin{aligned}
& \mathrm{P}_{\text {out }}^{D_{f}}=\operatorname{Pr}\left(\frac{a_{f} \gamma^{2} \rho_{S R} \rho_{R D_{f}}}{\left(a_{n}+d_{1}\right) \gamma^{2} \rho_{S R} \rho_{R D_{f}}+b_{1} \gamma \rho_{S R}+b_{2} \gamma \rho_{R D_{f}}+1}<\gamma_{\text {thf }}\right) \\
& =\operatorname{Pr}\left(\rho_{S R}<\frac{b_{2} c \gamma_{\text {thf }}}{a_{f}-a_{n} \gamma_{\text {thf }}-d_{1} \gamma_{\text {thf }}}=\lambda\right)+\operatorname{Pr}\left(\rho_{R D_{f}}<\frac{\lambda\left(b_{1} \rho_{S R}+c\right)}{b_{2}\left(\rho_{S R}-\lambda\right)}, \rho_{S R}>\lambda\right) \\
& =\operatorname{Pr}\left(\rho_{S R}<\lambda\right)+\int_{\lambda}^{\infty} f_{\rho_{S R}}(y)\left[1-\sum_{g_{R D_{f}}=0}^{\alpha_{R D_{f}}-1} \frac{1}{g_{R D_{f}} !} e^{-\frac{\lambda\left(b_{2} y+c\right)}{b_{2} \beta_{R f_{f}}(y-\lambda)}}\left(\frac{\lambda\left(b_{1} y+c\right)}{b_{2} \beta_{R D_{f}}(y-\lambda)}\right)^{g_{R D_{f}}}\right] d y \\
& =1-\frac{1}{\Gamma\left(\alpha_{S R}\right) \beta_{S R}^{\alpha_{S R}}} e^{-\frac{\lambda}{\beta_{S R}}-\frac{b_{1} \lambda}{b_{2} R_{R_{f}}}} \sum_{g_{R D_{f}}=0}^{\alpha_{R D_{f}}-1} \sum_{j=0}^{\alpha_{S R}-1} \sum_{n=0}^{g_{R D_{f}}}\left(\begin{array}{c}
\alpha_{S R}-1 \\
j
\end{array}\right)\left(\begin{array}{c}
g_{R D_{f}} \\
n
\end{array}\right)\left(\frac{\lambda}{b_{2} \beta_{R D_{f}}}\right)^{g_{R D_{f}}} \frac{\lambda^{\alpha_{S R}-1-j}}{g_{R D_{f}} !} \\
& \left(b_{1} \lambda+c\right)^{n}\left(b_{1}\right)^{g_{R D_{f}}-n} \underbrace{\int_{0}^{\infty} y^{j-n} e^{-\frac{y}{\beta_{S R}}-\frac{\lambda\left(b_{1} \lambda+c\right)}{b_{2} \beta_{R D_{f}} y}} d y}_{1_{1}}
\end{aligned}
$$

where $c=1 / \gamma, \mathrm{P}_{\text {out }}^{D_{f}}$ is based on the condition of $\gamma_{t h f}<a_{f} /\left(a_{n}+d_{1}\right)$. (A.1) follows Binomial 
theorem, $\mathrm{I}_{1}$ is obtained by using [Eq.(3.471.9)] in [25], so $\mathrm{I}_{1}$ can be rewritten as

$$
\mathrm{I}_{1}=2\left[\frac{\lambda \beta_{S R}\left(b_{1} \lambda+c\right)}{b_{2} \beta_{R D_{f}}}\right]^{\frac{j-n+1}{2}} K_{j-n+1}\left[2 \sqrt{\frac{\lambda\left(b_{1} \lambda+c\right)}{b_{2} \beta_{S R} \beta_{R D_{f}}}}\right]
$$

Substituting (A.2) into (A.1), we can obtain (15).

\section{APPENDIX B \\ PROOF OF THEOREM}

Substituting (7), (8), (12) and (13) into (17), the outage probability of $D_{n}$ is expressed as

$$
\begin{aligned}
& \mathrm{P}_{\text {out }}^{D_{n}}=\underbrace{\left[1-\operatorname{Pr}\left(\frac{a_{f} \gamma \rho_{S D_{n}}}{\left(a_{n}+\kappa_{S D_{n}}^{2}\right) \gamma \rho_{S D_{n}}+1} \geq \gamma_{t h f}, \frac{a_{n} \gamma \rho_{S D_{n}}}{\kappa_{S D_{n}}^{2} \gamma \rho_{S D_{n}}+1} \geq \gamma_{t h n}\right)\right]}_{\mathrm{I}_{2}}
\end{aligned}
$$

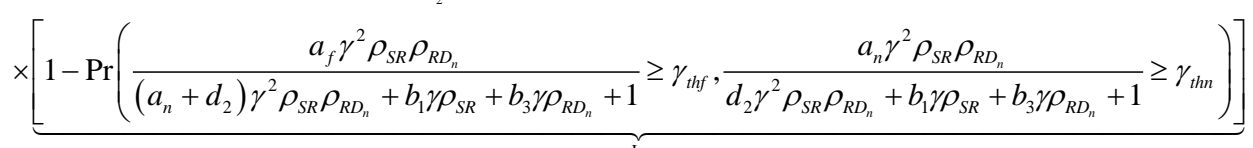

$\mathrm{I}_{2}$ and $\mathrm{I}_{3}$ are calculated as follows:

$$
\begin{aligned}
& \mathrm{I}_{2}=1-\operatorname{Pr}\left(\rho_{S D_{n}} \geq \frac{\gamma_{t h f} c}{a_{f}-\left(a_{n}+\kappa_{S D_{n}}^{2}\right) \gamma_{t h f}}=\tau_{1}\right) \operatorname{Pr}\left(\rho_{S D_{n}} \geq \frac{\gamma_{t h n} c}{a_{n}-\kappa_{S D_{n}}^{2} \gamma_{t h n}}=\tau_{2}\right) \\
& =1-\operatorname{Pr}\left(\rho_{S D_{n}} \geq \max \left(\tau_{1}, \tau_{2}\right)=\tau\right) \\
& =1-\sum_{g_{S D_{n}}=0}^{\alpha_{S D_{n}}-1} \frac{1}{g_{S D_{n}}} e^{-\frac{\tau}{\beta_{S D_{n}}}}\left(\frac{\tau}{\beta_{S D_{n}}}\right)^{g_{S D_{n}}} \\
& \mathrm{I}_{3}=1-\operatorname{Pr}\left(\rho_{R D_{n}} \geq \frac{\theta_{1}\left[b_{1} \rho_{S R}+c\right]}{b_{3}\left(\rho_{S R}-\theta_{1}\right)}, \rho_{S R} \geq \frac{b_{3} \gamma_{t h f}}{a_{1} \gamma-\left(a_{2}-d_{2}\right) \gamma \gamma_{t h f}}=\theta_{1}\right) \\
& \times \operatorname{Pr}\left(\rho_{R D_{n}} \geq \frac{\theta_{2}\left[b_{1} \rho_{S R}+c\right]}{b_{3}\left(\rho_{S R}-\theta_{2}\right)}, \rho_{S R} \geq \frac{b_{3} \gamma_{\text {thn }}}{a_{2} \gamma-d_{2} \gamma \gamma_{\text {thn }}}=\theta_{2}\right) \\
& =1-\operatorname{Pr}\left(\rho_{R D_{n}} \geq \frac{\theta\left[b_{1} \rho_{S R}+c\right]}{b_{3}\left(\rho_{S R}-\theta\right)}, \rho_{S R} \geq \max \left(\theta_{1}, \theta_{2}\right)=\theta\right) \\
& =1-\frac{1}{\Gamma\left(\alpha_{S R}\right) \beta_{S R}^{\alpha_{S R}}} e^{-\frac{\theta}{\beta_{S R}}-\frac{b_{1} \theta}{b_{3} R_{R D_{n}}}} \sum_{g_{R D_{n}}=0}^{\alpha_{R D_{n}}-1} \sum_{j=0}^{\alpha_{S R}-1} \sum_{n=0}^{g_{R D_{n}}}\left(\begin{array}{c}
\alpha_{S R}-1 \\
j
\end{array}\right)\left(\begin{array}{c}
g_{R D_{n}} \\
n
\end{array}\right)\left(\frac{\theta}{b_{3} \beta_{R D_{n}}}\right)^{g_{R D_{n}}} \\
& \times \frac{\theta^{\alpha_{S R}-1-j}}{g_{R D_{n}} !}\left(b_{1} \theta+c\right)^{n} \times\left(b_{1}\right)^{g_{R D_{n}}-n} \underbrace{\underbrace{\infty}_{0} y^{j-n} e^{-\frac{y}{\beta_{S R}}-\frac{\theta\left(b_{1} \theta+c\right)}{b_{3} \beta_{R D_{n} y} y}} d y}_{\mathrm{I}_{4}}
\end{aligned}
$$

$\mathrm{I}_{4}$ is obtained by using [Eq.(3.471.9)] in [25], so $\mathrm{I}_{4}$ can be rewritten as

$$
\mathrm{I}_{4}=2\left[\frac{\theta \beta_{S R}\left(b_{1} \theta+c\right)}{b_{3} \beta_{R D_{n}}}\right]^{\frac{j-n+1}{2}} \mathrm{~K}_{j-n+1}\left[2 \sqrt{\frac{\theta\left(b_{1} \theta+c\right)}{b_{3} \beta_{S R} \beta_{R D_{n}}}}\right]
$$

Substituting (B.4) into (B.3), then substitute (B.3) and (B.2) into (B.1), we can obtain (18).

\section{APPENDIX C PROOF OF COROLLARY 1}

Based on (14), a high-SNR approximation of $D_{f}$ is defined as 


$$
\begin{aligned}
& \mathrm{P}_{D_{f}}^{\infty}= \operatorname{Pr}\left(\frac{a_{f} \gamma^{2} \rho_{S R} \rho_{R D_{f}}}{\left(a_{n}+d_{1}\right) \gamma^{2} \rho_{S R} \rho_{R D_{f}}+b_{1} \gamma \rho_{S R}+b_{2} \gamma \rho_{R D_{f}}+1}<\gamma_{t h f}\right) \\
&= \operatorname{Pr}\left(\rho_{S R}<\frac{b_{2} c \gamma_{t h f}}{a_{f}-a_{n} \gamma_{t h f}-d_{1} \gamma_{t h f}}=\lambda\right)+\operatorname{Pr}\left(\rho_{R D_{f}}<\frac{\lambda\left(b_{1} \rho_{S R}+c\right)}{b_{2}\left(\rho_{S R}-\lambda\right)}, \rho_{S R}>\lambda\right) \\
&= \operatorname{Pr}\left(\rho_{S R}<\lambda\right)+\int_{\lambda}^{\infty} f_{\rho_{S R}}(y) \int_{0}^{\frac{\lambda\left(b_{2} y+c\right)}{b_{2}(y-\lambda)}} f_{\rho_{R D_{f}}}(x) d x d y \\
&= \frac{1}{\alpha_{S R} !}\left(\frac{\lambda}{\beta_{S R}}\right)^{\alpha_{S R}}+\frac{1}{\Gamma\left(\alpha_{S R}\right) \beta_{S R}^{\alpha_{S R}} \alpha_{R D_{f}} !} \sum_{j=0}^{\alpha_{R D_{f}}}\left(\begin{array}{c}
\alpha_{R D_{f}} \\
j
\end{array}\right)\left(\frac{\lambda}{b_{2} \beta_{R D_{f}}}\right)^{\alpha_{R D_{f}}} \\
& \times\left(b_{1} \lambda+c\right)^{j} b_{1}^{\alpha_{R D_{f}}-j} \underbrace{\int_{0}^{\infty}(y+\lambda)^{\alpha_{S R}-1} y^{-n} e^{-\frac{y+\lambda}{\beta_{S R}}} d y}_{\mathrm{I}_{\mathrm{I}}}
\end{aligned}
$$

Note that $I_{5}$ is supposed to the classical integration formulas in (C.2) when $\gamma \rightarrow \infty$.

$$
\mathrm{I}_{6}=\int_{0}^{\infty} y^{\alpha_{S R}-1-n} e^{-\frac{y}{\beta_{S R}}} d y
$$

Taking integer values on [25], we can obtain the $\mathrm{I}_{7}$ as follows:

$$
\mathrm{I}_{7}=\left(\alpha_{S R}-1-j\right) ! \beta_{S R}^{\alpha_{S R}-j}
$$

Substituting (C.3) into (C.1), we can obtain (21).

\section{APPENDIX D \\ PROOF OF COROLLARY 2}

Based on (17), a high-SNR approximation of $\mathrm{I}_{2}$ and $\mathrm{I}_{3}$ are defined as

$$
\begin{aligned}
& \mathrm{I}_{2}==1-\operatorname{Pr}\left(\rho_{S D_{n}} \geq \frac{\gamma_{t h f}}{a_{f} \gamma-\left(a_{n}+\kappa_{S D_{n}}^{2}\right) \gamma \gamma_{t h f}}=\tau_{1}\right) \operatorname{Pr}\left(\rho_{S D_{n}} \geq \frac{\gamma_{t h n}}{a_{n} \gamma-\kappa_{S D_{n}}^{2} \gamma \gamma_{t h n}}=\tau_{2}\right) \\
& =1-\operatorname{Pr}\left(\rho_{S D_{n}} \geq \max \left(\tau_{1}, \tau_{2}\right)=\tau\right) \\
& \approx \frac{1}{\alpha_{S D_{n}} !}\left(\frac{\tau}{\beta_{S D_{n}}}\right)^{\alpha_{S D_{n}}} \\
& \mathrm{I}_{3}=1-\operatorname{Pr}\left(\rho_{R D_{n}} \geq \frac{\theta_{1}\left[b_{1} \rho_{S R}+c\right]}{b_{3}\left(\rho_{S R}-\theta_{1}\right)}, \rho_{S R} \geq \frac{b_{3} c \gamma_{\text {thf }}}{a_{1}-\left(a_{2}-d_{2}\right) \gamma_{\text {thf }}}=\theta_{1}\right) \\
& \times \operatorname{Pr}\left(\rho_{R D_{n}} \geq \frac{\theta_{2}\left[b_{1} \rho_{S R}+c\right]}{b_{3}\left(\rho_{S R}-\theta_{2}\right)}, \rho_{S R} \geq \frac{b_{3} c \gamma_{t h n}}{a_{2}-d_{2} \gamma_{t h n}}=\theta_{2}\right) \\
& =1-\operatorname{Pr}\left(\rho_{R D_{n}} \geq \frac{\theta\left[b_{1} \rho_{S R}+c\right]}{b_{3}\left(\rho_{S R}-\theta\right)}, \rho_{S R} \geq \max \left(\theta_{1}, \theta_{2}\right)=\theta\right) \\
& =\int_{0}^{\infty} f_{\rho_{S R}}(y+\theta) \frac{1}{\alpha_{R D_{n}} !}\left(\frac{\theta\left(b_{1} y+b_{1} \theta+c\right)}{b_{3} \beta_{R D_{n}} y}\right)^{\alpha_{R D_{n}}} d y \\
& =\frac{1}{\Gamma\left(\alpha_{S R}\right) \beta_{S R}^{\alpha_{S R}} \alpha_{R D_{n}} !} \sum_{j=0}^{\alpha_{R D_{n}}}\left(\begin{array}{c}
\alpha_{R D_{n}} \\
j
\end{array}\right)\left(\frac{\theta}{b_{3} \beta_{R D_{n}}}\right)^{\alpha_{R D_{n}}}\left(b_{1} \theta+c\right)^{j} \\
& \times b_{1}^{\alpha_{\text {SOn }}-j} \underbrace{\int_{0}^{\infty}(y+\theta)^{\alpha_{\mathrm{SR}}-1} y^{-n} e^{\frac{-y+\theta}{\beta_{S R}}} d y}_{\mathrm{I}_{6}}
\end{aligned}
$$

Note that $I_{6}$ is supposed to the classical untegration formulas in (D.2) when $\gamma \rightarrow \infty$, and it is lucky to get a formula equals to the (C.2). Therefore, substituting (C.3) into (D.2), then (22) is 
obtained by combining (D.1) and (D.2).

\section{APPENDIX E \\ PROOF OF COROLLARY 3}

At high SNRs $(\gamma \rightarrow \infty)$, the asymptotic ergodic sum rate $R_{\text {ave }}^{D_{f}}$ and $R_{\text {ave }}^{D_{n}}$ can be rewritten as

$$
\begin{aligned}
R_{\text {ave }}^{D_{f}, n i} & =\frac{1}{2} \log _{2}\left(1+\frac{a_{f} \gamma^{2} \rho_{S R} \rho_{R D_{f}}}{\left(a_{n}+d_{1}\right) \gamma^{2} \rho_{S R} \rho_{R D_{f}}+b_{1} \gamma \rho_{S R}+b_{2} \gamma \rho_{R D_{f}}+1}\right) \\
& \approx \frac{1}{2} \log _{2}\left(1+\frac{a_{f}}{a_{n}+d_{1}}\right) \\
R_{\text {ave }}^{D_{n}, n i} & =\frac{1}{2} \log _{2}\left(1+\max \left[\frac{a_{n} \gamma \rho_{S D_{n}}}{\kappa_{S D_{n}}^{2} \gamma \rho_{S D_{n}}+1}, \frac{a_{n} \gamma^{2} \rho_{S R} \rho_{R D_{n}}}{d_{2} \gamma^{2} \rho_{S R} \rho_{R D_{n}}+b_{1} \gamma \rho_{S R}+b_{3} \gamma \rho_{R D_{n}}+1}\right]\right) \\
\approx & \frac{1}{2} \log _{2}\left(1+\max \left[\frac{a_{n}}{\kappa_{S D_{n}}^{2}}, \frac{a_{n}}{d_{2}}\right]\right) \\
\approx & \frac{1}{2} \log _{2}\left(1+\frac{a_{n}}{\hat{k}}\right)
\end{aligned}
$$

where $\hat{k}=\min \left[\kappa_{S D_{n}}^{2}, d_{2}\right]$

$$
\begin{aligned}
R_{\text {ave }}^{D_{n, 2}, n i} & =\frac{1}{2} \log _{2}\left(1+\frac{a_{n} \gamma \rho_{S D_{n}}}{\kappa_{S D_{n}}^{2} \gamma \rho_{S D_{n}}+1}\right) \\
& \approx \frac{1}{2} \log _{2}\left(1+\frac{a_{2}}{\kappa_{S D_{n}}^{2}}\right)
\end{aligned}
$$

Finally, combining (E.1), (E.2) and (E.3), the asymptotic ergodic sum rate can be derived as (31).

\section{APPENDIX F}

PROOF OF COROLLARY 4

In the high-SNR regime, we have

$$
\begin{aligned}
R_{\text {ave }}^{D_{f}, i d} & =\frac{1}{2} \log _{2}\left(1+\frac{a_{f} \gamma^{2} \rho_{S R} \rho_{R D_{f}}}{a_{n} \gamma^{2} \rho_{S R} \rho_{R D_{f}}+\gamma \rho_{S R}+\gamma \rho_{R D_{f}}+1}\right) \\
& \approx \frac{1}{2} \log _{2}\left(1+\frac{a_{f}}{a_{n}}\right) \\
R_{\text {ave }}^{D_{n, 1}, i d}= & \mathrm{E}\left\{\frac{1}{2} \log _{2}\left(1+\max \left[a_{n} \gamma \rho_{S D_{n}}, \frac{a_{n} \gamma^{2} \rho_{S R} \rho_{R D_{n}}}{\gamma \rho_{S R}+\gamma \rho_{R D_{n}}+1}\right]\right)\right\} \\
\leq & \mathrm{E}\left\{\frac{1}{2 \ln 2} \ln \left(1+a_{n} \gamma \max \left[\rho_{S D_{n}}, \min \left[\rho_{S R}, \rho_{R D_{n}}\right]\right]\right)\right\}
\end{aligned}
$$

where $R_{a v e}^{D_{n, 1}, i d}$ is obtained by the inequality [24]

$$
\frac{\gamma^{2} \rho_{S R} \rho_{R D_{n}}}{\gamma \rho_{S R}+\gamma \rho_{R D_{n}}+1} \leq \min \left[\gamma \rho_{S R}, \gamma \rho_{R D_{n}}\right]
$$

Denote $W=\max \left[\rho_{S D_{n}}, \min \left(\rho_{S R}, \rho_{R D_{n}}\right)\right]$, the upper bound for $R_{\text {ave }}^{D_{n,}, i d}$ can be calculated as 


$$
\begin{aligned}
R_{\text {ave }}^{D_{2,1}, i d} & \leq \mathrm{E}\left[\frac{1}{2 \ln 2} \ln \left(1+a_{n} \gamma w\right)\right] \\
& =\frac{1}{2 \ln 2} \int_{o}^{\infty} f_{w}(w) \ln \left(1+a_{n} \gamma w\right) d w \\
& =\frac{a_{n} \gamma}{2 \ln 2} \int_{0}^{\infty} \frac{1-F_{W}(w)}{1+a_{n} \gamma w} d w
\end{aligned}
$$

To approximate the ultimate expression, we need to calculate the CDF of $W$, which is given by the following analysis.

$$
\begin{aligned}
& F_{W}(w)=\operatorname{Pr}\left(\max \left[\rho_{S D_{n}}, \min \left(\rho_{S R}, \rho_{R D_{n}}\right)\right] \leq w\right) \\
& =\operatorname{Pr}\left(\rho_{S D_{n}} \leq w\right)\left[1-\operatorname{Pr}\left(\rho_{S R}>w\right) \operatorname{Pr}\left(\rho_{R D_{n}}>w\right)\right] \\
& =1-\sum_{g_{S D_{n}}=0}^{\alpha_{S D_{n}}-1} \frac{1}{g_{S D_{n}}} e^{-\frac{w}{\beta_{S D_{n}}}}\left(\frac{w}{\beta_{S D_{n}}}\right)^{g_{S D_{n}}}-\sum_{g_{S R}=0}^{\alpha_{S R}-1} \sum_{g_{R D_{n}}=0}^{\alpha_{R_{D_{n}}}-1} \frac{1}{g_{S R} ! g_{R D_{n}} !} e^{-\frac{w}{\beta_{S R}}-\frac{w}{\beta_{R D_{n}}}} \\
& \times\left(\frac{w}{\beta_{S R}}\right)^{g_{S R}}\left(\frac{w}{\beta_{R D_{n}}}\right)^{g_{R D_{n}}}+\sum_{g_{S R}=0}^{\alpha_{S R}-1} \sum_{g_{R D_{n}}=0}^{\alpha_{R D_{n}}-1} \sum_{g_{S D_{n}}=0}^{\alpha_{S D_{n}}-1} \frac{1}{g_{S R} ! g_{R D_{n}} ! g_{S D_{n}} !} \\
& \times e^{-\frac{w}{\beta_{S D_{n}}}-\frac{w}{\beta_{S R}}-\frac{w}{\beta_{R D_{n}}}}\left(\frac{w}{\beta_{S R}}\right)^{g_{S R}}\left(\frac{w}{\beta_{R D_{n}}}\right)^{g_{R D_{n}}}\left(\frac{w}{\beta_{S D_{n}}}\right)^{g_{S D_{n}}}
\end{aligned}
$$

It is noted that by substituting (F.5) into (F.4), and defining $v=a_{n} \gamma w, R_{a v e}^{D_{n, 1} \text { id }}$ can be attained as

$$
\begin{aligned}
& R_{a v e}^{D_{n, 1}, i d} \leq \frac{1}{2 \ln 2}\{\sum_{g_{S D_{n}}}^{\alpha_{S D_{0}}-1} \frac{1}{g_{S D_{n}} !}\left(\frac{1}{a_{n} \gamma \beta_{S D_{n}}}\right)^{g_{S D_{n}}} \underbrace{\int_{0}^{\infty} \frac{e^{-\frac{v}{a_{n} \gamma / S_{S D_{n}}}} v^{g_{S D_{n}}}}{1+v} d v}_{\Phi_{1}}+\sum_{g_{S R}=0}^{\alpha_{S R}-1 \alpha_{g_{R D_{n}}}=0} \\
& \times \frac{1}{g_{S R} ! g_{R D_{n}} !}\left(\frac{1}{a_{n} \gamma \beta_{S R}}\right)^{g_{S R}}\left(\frac{1}{a_{n} \gamma \beta_{R D_{n}}}\right)^{g_{R D_{n}}} \underbrace{\int_{0}^{\infty} \frac{e^{-\frac{v}{a_{n} \gamma \beta_{S R}}-\frac{v}{a_{n} \gamma \beta_{R D_{n}}}} v^{g_{S R}+g_{R D_{n}}}}{1+v} d v}_{\Phi_{2}} \\
& +\sum_{g_{S R}=0}^{\alpha_{S R}-1} \sum_{g_{R D_{n}}=0}^{\alpha_{R D_{n}}-1} \sum_{\alpha_{S D_{n}}=0} \frac{1}{g_{S R} ! g_{R D_{n}} ! g_{S D_{n}} !}\left(\frac{1}{a_{n} \gamma \beta_{S R}}\right)^{g_{S R}}\left(\frac{1}{a_{n} \gamma \beta_{R D_{n}}}\right)^{g_{R D_{n}}} \\
& \times\left(\frac{1}{a_{n} \gamma \beta_{S D_{n}}}\right)^{g_{S D_{n}}} \underbrace{\int_{0}^{\infty} \frac{e^{-\frac{v}{a_{n} \gamma \beta S_{S R}}-\frac{v}{a_{n} \gamma \beta \beta_{D_{n}}}-\frac{v}{a_{n} \beta_{S D_{n}}}} v_{S S_{R}+g_{R D_{n}}+g_{S D_{n}}}}{1+v} d v}_{\Phi_{3}}\}
\end{aligned}
$$

Defining $f_{1}=1 / a_{n} \gamma \beta_{S D_{n}}, f_{2}=1 / a_{n} \gamma \beta_{S R}, f_{3}=1 / a_{n} \gamma \beta_{R D_{n}}$, then with the aid of [25] [Eq. (3.352.4)] and [Eq.(3.353.5. ${ }^{7}$ ], we can arrive the expressions of $\Phi_{1}, \Phi_{2}$ and $\Phi_{3}$.

$$
\begin{aligned}
& \Phi_{1}=\left\{\begin{array}{l}
-e^{f_{1}} \operatorname{Ei}\left(-f_{1}\right), \quad g_{S D_{n}}=0 \\
(-1)^{g_{S D_{n}}-1} e^{f_{1}} \operatorname{Ei}\left(-f_{1}\right)+\sum_{L=1}^{g_{S D_{n}}}(L-1) !(-1)^{g_{S D_{n}}-L}\left(f_{1}\right)^{-L}, \quad g_{S D_{n}}>0
\end{array}\right. \\
& \Phi_{2}=\left\{\begin{array}{cc}
-e^{f_{1}+f_{3}} \operatorname{Ei}\left(-f_{2}-f_{3}\right), \quad g_{S R}+g_{R D_{n}}=0 \\
(-1)^{g_{S R}+g_{R D_{n}}-1} e^{f_{2}+f_{3}} \operatorname{Ei}\left(-f_{2}-f_{3}\right)+\sum_{L=1}^{g_{S R}+g_{R D_{n}}}(L-1) ! \\
\quad \times(-1)^{g_{S R}+g_{R D_{n}}-L}\left(f_{2}+f_{3}\right)^{-L}, \quad & g_{S R}+g_{R D_{n}}>0
\end{array}\right.
\end{aligned}
$$




$$
\Phi_{3}=\left\{\begin{array}{l}
-e^{f_{1}+f_{2}+f_{3}} \operatorname{Ei}\left(-f_{1}-f_{2}-f_{3}\right), \quad g_{S R}+g_{R D_{n}}+g_{S D_{n}}=0 \\
(-1)^{g_{S R}+g_{R D_{n}}+g_{S D_{n}}-1} e^{f_{1}+f_{2}+f_{3}} \operatorname{Ei}\left(-f_{1}-f_{2}-f_{3}\right)+\sum_{L=1}^{g_{S R}+g_{R D_{n}}+g_{S D_{n}}}(L-1) ! \\
\quad \times(-1)^{g_{S R}+g_{R D_{n}}+g_{S D_{n}}-L}\left(f_{1}+f_{2}+f_{3}\right)^{-L}, \quad g_{S R}+g_{R D_{n}}+g_{S D_{n}}>0
\end{array}\right.
$$

Next, we note that $R_{\text {ave }}^{D_{2,2}, \text { id }}$ can be expressed as follows:

$$
R_{\text {ave }}^{D_{n, 2} \text { id }}=\mathrm{E}\left[\frac{1}{2} \log _{2}\left(1+a_{n} \gamma \rho_{S D_{n}}\right)\right]
$$

Denote $W=\rho_{S D_{n}}$, and $W$ follows the Nakagami- $m$ distribution , the CDF of $W$ turns out to be

$$
\begin{aligned}
F_{W}(w) & =\operatorname{Pr}\left(\rho_{S D_{n}} \leq w\right) \\
& =1-\sum_{g_{S D_{n}}=0}^{\alpha_{S D_{n}}-1} \frac{1}{g_{S D_{n}} !} e^{-w / \beta_{S D_{n}}}\left(\frac{w}{\beta_{S D_{n}}}\right)^{g_{S D_{n}}}
\end{aligned}
$$

Therefore, $R_{\text {ave }}^{D_{\text {ne, }} \text { id }}$ can be rewritten as

$$
\begin{aligned}
R_{\text {ave }}^{D_{n, 2}, i d} & =\frac{a_{n} \gamma}{2 \ln 2} \int_{0}^{\infty} \frac{1-F_{W}(w)}{1+a_{2} \gamma w} d w \\
& =\sum_{g_{S D_{n}}}^{\alpha_{S D_{n}}-1} \frac{1}{g_{S D_{n}} !}\left(\frac{1}{a_{n} \gamma \beta_{S D_{n}}}\right)^{g_{4}} \underbrace{\int_{0}^{\infty} \frac{e^{-\frac{v}{a_{n} \gamma \beta_{S D_{n}}}} v^{g_{S D_{n}}}}{1+v} d v}_{\Phi_{1}}
\end{aligned}
$$

According to (F.7), $R_{a v e}^{D_{\text {nve }}, \text { id }}$ is obtained. Finally, combine (F.1), (F.6) and (F.12), we can acquire the upper bound for ergodic sum rate of ideal hardware in (32).

\section{References}

[1] Q. C. Li, H. Niu, A. T. Papathanassiou and G. Wu, "5G Network Capacity: Key Elements and Technologies,” IEEE Veh. Technol. Mag., vol. 9, no. 1, pp. 71-78, Mar. 2014.

Article (CrossRef Link)

[2] X. Li, X. Yang, L. Li, J. Jin, N. Zhao and C. Zhang, "Performance Analysis of Distributed MIMO With ZF Receivers Over Semi-Correlated K Fading Channels,” IEEE Access, vol. PP, no. 99, pp. 1-1, Jun. 2017. Article (CrossRef Link)

[3] H. Sun, Y. Xu and R. Q. Hu, "A NOMA and MU-MIMO Supported Cellular Network with Underlaid D2D Communications," in Proc. of 2016 IEEE 83rd Vehicular Technology Conference (VTC Spring), Nanjing, 2016, pp. 1-5. Article (CrossRef Link)

[4] S. F. Yunas, M. Valkama and J. Niemelä, "Spectral and energy efficiency of ultra-dense networks under different deployment strategies,” IEEE Commun. Mag., vol. 53, no. 1, pp. 90-100, Jan. 2015. Article (CrossRef Link)

[5] X. Li, J. Li, L. Li, J. Jin, L. Du and D. Zhang, "Performance Analysis of Cooperative Small-Cell Networks Under Correlated Rician/Gamma Fading Channels,” IET Signal Process., 2017.

Article (CrossRef Link)

[6] J. Zhang, X. Yang, Q. Yao, X. Ge, M. Jo and G. Mao, "Cooperative Energy Efficiency Modeling and Performance Analysis in Co-Channel Interference Cellular Networks," Computer Journal, vol. 56, no. 8, pp. 1010-1019, Aug. 2013. Article (CrossRef Link)

[7] Z. Zhang, H. Sun and R. Q. Hu, "Downlink and Uplink Non-Orthogonal Multiple Access in a Dense Wireless Network," IEEE Journal on Selected Areas in Communications, vol. 35, no. 12, pp. 2771-2784, Dec. 2017. Article (CrossRef Link)

[8] H. Sun, B. Xie, R. Q. Hu and G. Wu, "Non-Orthogonal Multiple Access with SIC Error Propagation in Downlink Wireless MIMO Networks," in Proc. of 2016 IEEE 84th Vehicular Technology Conference (VTC-Fall), pp. 1-5, Sep. 2016. Article (CrossRef Link) 
[9] M. Xu, F. Ji, M. Wen and W. Duan, "Novel Receiver Design for the Cooperative Relaying System with Non-Orthogonal Multiple Access,” IEEE Commun. Lett., vol. 20, no. 8, pp. 1679-1682, Aug. 2016. (CrossRef Link)

[10] J. B. Kim and I. H. Lee, "Capacity Analysis of Cooperative Relaying Networks Using Non-Orthogonal Multiple Access,” IEEE Commun. Lett., vol. 19, no. 11, pp. 1949-1952, Nov. 2015. Article (CrossRef Link)

[11] M. F. Kader and S. Y. Shin, "Exploiting cooperative diversity with non-orthogonal multiple access over slow fading channel," International Journal of Electronics, vol. 104, no. 6, pp. 1050-1062, 2017. Article (CrossRef Link)

[12] J. B. Kim and I. H. Lee, "Non-Orthogonal Multiple Access in Coordinated Direct and Relay Transmission,” IEEE Commun. Lett., vol. 19, no. 11, pp. 2037-2040, Nov. 2015. Article (CrossRef Link)

[13] M. F. Kader and S. Y. Shin, "Cooperative Relaying Using Space-Time Block Coded Non-Orthogonal Multiple Access,” IEEE Trans. Veh. Technol., vol. 66, no. 7, pp. 5894-5903, Jul. 2017. Article (CrossRef Link)

[14] X. Yue, Y. Liu, S. Kang and A. Nallanathan, "Performance Analysis of NOMA With Fixed Gain Relaying Over Nakagami-m Fading Channels,” IEEE Access, vol. 5, pp. 5445-5454, Mar. 2017. Article (CrossRef Link)

[15] J. Men, J. Ge and C. Zhang, "Performance Analysis of Nonorthogonal Multiple Access for Relaying Networks Over Nakagami-m Fading Channels,” IEEE Trans. Veh. Technol., vol. 66, no. 2, pp. 1200-1208, Feb. 2017. Article (CrossRef Link)

[16] J. Men, J. Ge, and C. Zhang, "Performance Analysis for Downlink Relaying Aided Non-orthogonal Multiple Access Networks with Imperfect CSI over Nakagami-m Fading,” IEEE Access, vol. 5, pp. 998-1004, Nov. 2016. Article (CrossRef Link)

[17] T. Schenk, "RF Imperfections in High-rate Wireless Networks: Impact and Digital Compensation,” Springer Netherlands, 2008. Article (CrossRef Link)

[18] X. Li, J. Li, J. Jin and L. Li, "Performance analysis of relaying systems over Nakagami-m fading with transceiver hardware impairments," Journal of XIDIAN University., vol. 45, no. 3. 2018. Article (CrossRef Link)

[19] A. K. Papazafeiropoulos, S. K. Sharma, S. Chatzinotas and B. Ottersten, "Ergodic Capacity Analysis of AF DH MIMO Relay Systems With Residual Transceiver Hardware Impairments: Conventional and Large System Limits,” IEEE Trans. Veh. Technol., vol. 66, no. 8, pp. 7010-7025, Aug. 2017. Article (CrossRef Link)

[20] M. Matthaiou, A. Papadogiannis, E. Bjornson and M. Debbah, "Two-Way Relaying Under the Presence of Relay Transceiver Hardware Impairments,” IEEE Commun. Lett., vol. 17, no. 6, pp. 1136-1139, Jun. 2013. Article (CrossRef Link)

[21] E. Bjornson, M. Matthaiou and M. Debbah, "A New Look at Dual-Hop Relaying: Performance Limits with Hardware Impairments,” IEEE Trans. Commun., vol. 61, no. 11, pp. 4512-4525, Nov. 2013. Article (CrossRef Link)

[22] A. Cheaito, M. Crussière, J. F. Hélard and Y. Louët, "Quantifying the Memory Effects of Power Amplifiers: EVM Closed-Form Derivations of Multicarrier Signals,” IEEE Wireless Commun. Lett., vol. 6, no. 1, pp. 34-37, Feb. 2015. Article (CrossRef Link)

[23] M. M. Molu, P. Xiao, M. Khalily, L. Zhang and R. Tafazolli, “A Novel Equivalent Definition of Modified Bessel Functions for Performance Analysis of Multi-Hop Wireless Communication Systems,” IEEE Access, vol. 5, no. 99, pp. 7594-7605, May 2017. Article (CrossRef Link)

[24] J. Men and J. Ge, "Performance analysis of non-orthogonal multiple access in downlink cooperative network,” Commun. Iet, vol. 9, no. 18, pp. 2267-2273, Dec. 2015. Article (CrossRef Link)

[25] I. S. Gradshteyn and I. M. Ryzhik, "Table of Integrals, Series, and Products(Seventh Edition)," Academic Press, 2007. Article (CrossRef Link) 


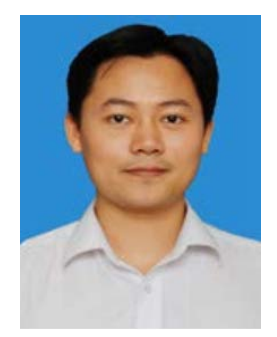

Chao Deng received the B.Sc. degree and the M. Sc. degree in communication engineering from Jilin University, China, in 2002 and 2005. He then received the Ph. D. degrees in Changchun Institute of Optics, Fine Mechanics and Physics, Chinese Academy of Sciences in 2008. He is currently an associate professor with the School of Physics and Electronic Information Engineering, Henan Polytechnic University, Jiaozuo China. His research interests include image processing and signal processing.

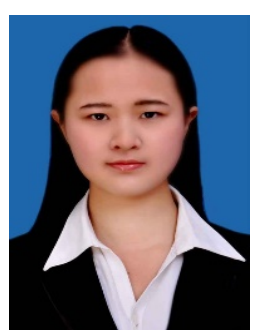

Xiaoya Zhao received the B.Sc degree in electronic information engineering from the College of Physics and Electronic Information, Xinyang Normal University, in 2016. She is currently pursuing the M.Sc. degree in communication and information systems with the School of Physics and Electronic Information Engineering, Henan Polytechnic University, Jiaozuo China. Her current research interests include nonorthogonal multiple access and hardware constrained communication.

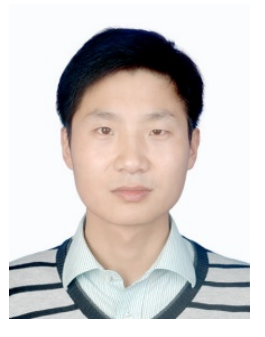

Di Zhang (SM'14) is currently pursuing the Ph.D. degree with Waseda University, Tokyo, Japan, since 2013. He served as the Workshop TPC Member of the IEEE ICC and the IEEE WCNC, and the IEEE VTC Reviewer of varies journals and conferences, such as IEEE Transactions on Wireless Communications, IEEE Wireless Communications Letters, IEEE Transactions on Vehicle Technology, IEEE WCNC, and IEEE VTC. He is a Student Member of IEICE. He received the Best Master Thesis, Distinction 1st honor, and other honors and grants. His current research interests include green communications, wireless communications, ICN, information theory, and signal processing.

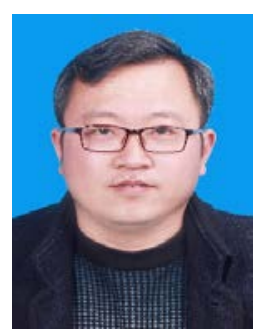

Xingwang $\mathbf{L i}$ received the B.Sc. degree in communication engineering from Henan Polytechnic University, China, in 2007. He then received the M. Sc. degree from the National Key Laboratory of Science and Technology on Communications at University of Electronic Science and Technology of China (UESTC) and Ph. D. degrees in communication and information system from the State Key Laboratory of Networking and Switching Technology at Beijing University of Posts and Telecommunications (BUPT). From 2017-2018, he was a Visiting Researcher Fellow with ECIT Institute, Queen's University Belfast, U.K. He is currently a lecturer in the Physics and Electronic Information Engineering, Henan Polytechnic University, Jiaozuo China. His research interests span massive MIMO, hardware constrained communication, physical layer security, nonorthogonal multiple access, FSO communications, and performance analysis of fading channels.

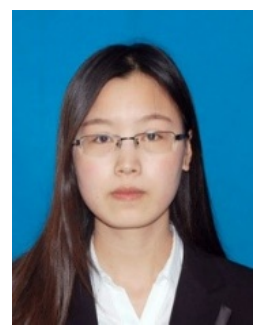

Jingjing Li received the B.Sc. degree in communication engineering from the College of Automation, Shenyang Institute of Engineering, in 2016. She is currently pursuing the M.Sc. degree in communication and information systems with the School of Physics and Electronic Information Engineering, Henan Polytechnic University, Jiaozuo China. Her current research interests include nonorthogonal multiple access and hardware constrained communication. 


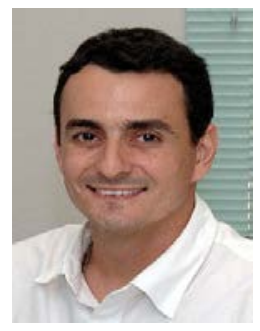

Charles Casimiro Cavalcante received the B.Sc and M.Sc in Electrical Engineering from the Federal University of Cear (UFC), Brazil, in 1999 and 2001, respectively, and the Ph.D. degree from the University of Campinas (UNICAMP), Brazil, in 2004. He has held a grant for Scientific and Technological Development from 2004 to 2007 and since March 2009 he has a grant of Scientific Research Productivity both from the Brazilian Research Council (CNPq). From March 2007 to November 2008 he was a Visiting Professor at Teleinformatics Engineering Department of UFC and since November 2008 he is an Assistant Professor at the same department and university holding the Statistical Signal Processing chair. From August 2014 to July 2015, he was a Visiting Assistent Professor at the Department of Computer Science and Electrical Engineering (CSEE) from University of Maryland, Baltimore County (UMBC) in the United States. He has been working on signal processing strategies for communications where he has several papers published in journals and conferences, has authored three international patents and he has worked on several funded research projects on the signal processing and wireless communications areas. He is also a co-author of the book Unsupervised Signal Processing: Channel Equalization and Source Separation, published by CRC Press. He is a researcher of the Wireless Telecommunications Research Group (GTEL) where he leads research on signal processing and wireless communications. Dr. Cavalcante is a Senior Member of the IEEE and a Senior Member of the Brazilian Telecommunications Society (SBrT). His main research interests are in signal processing for communications, blind source separation, wireless communications, and statistical signal processing. 